\title{
UN MÉTODO EFICIENTE PARA LA SIMULACIÓN DE CURVAS DE TASAS DE INTERÉS
}

\author{
Javier Márquez Diez-Canedo*
}

Banco de México

Carlos E. Nogués Nivón

Banco de México

Viviana Vélez Grajales

Banco de México

(Recibido 20 de febrero 2003, aceptado 28 de mayo 2003)

\begin{abstract}
Resumen
El objetivo de este trabajo es presentar una metodología para simular, con un mínimo de parámetros, estructuras temporales de tasas de interés que conserven la forma de las curvas que se construyen a partir de datos históricos observados. Esto se logra aprovechando la parsimonia del modelo de Nelson-Siegel, el cual puede reproducir la multiplicidad de formas de las curvas de tasas, observadas en la realidad, mediante el uso de sólo cuatro parámetros. Así, el proceso sólo requiere muestrear de forma aleatoria ocurrencias de los valores de estos cuatro parámetros; cualquier otro punto sobre la curva se obtiene directamente de la fórmula de Nelson-Siegel (1987). Para lograr lo anterior, trabajamos con las series históricas de los parámetros que generan dichas estructuras, asi como con su distribución de probabilidad conjunta. A partir de ésta, se pueden generar tanto series aleatorias de parámetros que sei comporten de acuerdo a la distribución histórica, como curvas de estructuras de tasas de interés por plazos correspondientes.
\end{abstract}

\section{Abstract}

In this paper, the authors present a methodology for efficiently simulating interest rate termstructure curves, with a minimum number of parameters. This is possible by taking advantage of Nelson and Siegel's model, which can reproduce the multiplicity of shapes commonly observed in historical data, using just four parameters. Thus, the process only requires the generation of random samples of four parameters to generate interest rate term-structure scenarios, and the nodes in the curve that are of particular interest are obtained by direct substitution into the Nelson-Siegel equation (1987). The paper shows how to simulate interest rate term-structure curves that behave in the same way as historically observed term-structure curves. The process requires series of parameters for Nelson-Siegel curves fitted to historical data, and their joint empirical probability distribution, which is then used to generate random series of parameters and the corresponding Nelson-Siegel curves.

Clasificación JEL: C15, E43

Palabras clave: Estructuras de Tasas de Interés, Simulación Monte Carlo, Nelson-Siegel

* Av. Cinco de Mayo No. 6, Colonia Centro, C.P. 06059 , México, D.F. Teléfono 52372000 .

Los autores desean agradecer a dos dictaminadores anónimos sus valiosas observaciones. Las opiniones expresadas en este documento son responsabilidad exclusiva de los autores y no necesariamente corresponden a las del Banco de México. 


\section{Introducción}

La medición del riesgo de mercado de un portafolio de activos financieros se estima a partir de los cambios en su valor ocasionados por movimientos en los factores de riesgo. En el caso de instrumentos de deuda, el factor de riesgo más importante es la tasa de interés. Además, también es un factor de riesgo útil en la valuación de otros instrumentos, como pueden ser las opciones, los futuros y los forwards.

Tratándose de bonos, el método de valuación usual es el de descontar a valor presente los flujos futuros que generan; para lo cual es necesario conocer dichos flujos, sus fechas de pago y los factores de descuento que se deben aplicar a esos flujos. Sin embargo, mientras que los flujos y las fechas de pago se especifican en cada contrato, los factores de descuento son el resultado de un consenso de mercado, respecto a la estructura temporal de tasas de interés futura que aplique a la valuación del bono. Como esta estructura es incierta, con frecuencia se recurre a la simulación Monte Carlo para realizar análisis de riesgo en carteras de bonos. Una razón por la cuál se emplea este método, es que con él es posible generar una multiplicidad de estructuras temporales de tasas de interés y obtener así el perfil de riesgo de la cartera y su VaR. ${ }^{1}$

Sin embargo, es común que dicha simulación se haga fijando de antemano el número de puntos (plazos) de la curva y simulando punto por punto. Esta tarea resulta ser difícil, puede producir resultados incongruentes y generalmente requiere de un código complicado y un esfuerzo computacional exagerado. Este artículo examina el uso del modelo de Nelson-Siegel para generar curvas de tasas, muestreando aleatoriamente sólo cuatro parámetros; lo cual significa una reducción en el esfuerzo con relación al número de puntos sobre la curva que se requieren simular de acuerdo con el método de Monte Carlo. ${ }^{2}$

\section{Tasas spot y tasas forward ${ }^{3}$}

Antes de entrar formalmente en materia, en esta sección se revisan las expresiones algebraicas que relacionan a las tasas spot con las tasas forward. Debido a que el manejo algebraico de las tasas anualmente compuestas es más complejo, a lo largo de esta sección se manejarán tasas compuestas en forma continua.

Los estudios de tasas de interés a distintos plazos, se realizan normalmente a partir de la información contenida en instrumentos de deuda o bonos. En teoría, bajo el supuesto de un mercado completo y sin impuestos, el precio de un bono es el valor presente de los cupones que paga más el valor presente del principal. Las tasas de descuento a distintos plazos utilizadas en el cálculo, corresponden a una estructura temporal de tasas spot. Es decir, la estructura de tasas a una fecha dada $t$ se representa por una gráfica de la tasa spot para cada fecha de vencimiento. ${ }^{4}$

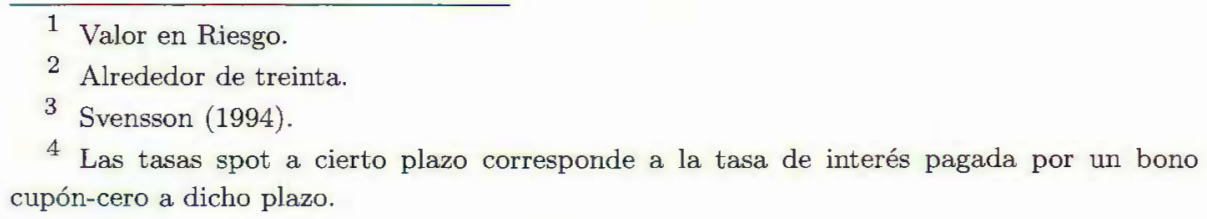


Sea $r(t, T)$ la tasa de interés compuesta continuamente de un bono cupóncero que se negocia en el momento $t$ y vence en el tiempo $T$, donde $T>t$. Sea $m=T-t$ el tiempo que falta para el vencimiento. El precio al tiempo $t$ de este bono cupón-cero con principal igual a 1 peso, denotará la función de descuento $\mathrm{d}(t, T)$. Así, la función de descuento y la tasa spot se relacionan de la siguiente manera:

$$
\mathrm{d}(t, T)=P(t, T)=e^{-r(t, T)(T-t)} .
$$

Ahora considérese un bono con valor nominal de 100 pesos que paga cupones anuales de $c$ pesos y vence dentro de $m$ años. El valor presente en $t$ del pago del cupón realizado el año $k, k=1,2, \ldots . ., m$, será $c \times \mathrm{d}(t, t+k)$ y el valor presente del principal que se pagará el año $m$ será $100 \times \mathrm{d}(t, t+m)$. De tal manera que el precio del bono el día de la transacción será:

$$
P(t, t+m)=\sum_{k=1}^{m} c \mathrm{~d}(t, t+k)+100 \mathrm{~d}(t, t+m) .
$$

Sea $f\left(t, t^{\prime}, T\right)$ la tasa forward continuamente compuesta de un contrato forward negociado en $t$ y cuya operación subyacente inicia en $t^{\prime}$ y vence en $T$, donde $T>t^{\prime}>t$. Entonces la tasa forward se relaciona con la tasa spot de la siguiente manera:

$$
f\left(t, t^{\prime}, T\right)=\frac{(T-t) r(t, T)-\left(t^{\prime}-t\right) r\left(t, t^{\prime}\right)}{T-t^{\prime}} .
$$

La tasa forward instantánea, es la tasa forward de un contrato que vence en un periodo infinitesimal y se define como sigue:

$$
f\left(t, t^{\prime}\right)=\lim _{T \rightarrow t^{\prime}} f\left(t, t^{\prime}, T\right)
$$

La tasa forward $f\left(t, t^{\prime}, T\right)$ es el promedio de las tasas forward instantáneas:

$$
f\left(t, t^{\prime}, T\right)=\frac{\int_{u=t^{\prime}}^{T} f(t, u) \mathrm{d} u}{T-t^{\prime}} .
$$

De igual forma, la tasa spot $r(t, T)$ al tiempo $t$ y plazo $T$ es el promedio de las tasas forward instantáneas:

$$
r(t, T)=\frac{\int_{u=t}^{T} f(t, u) \mathrm{d} u}{T-t} .
$$

\section{El modelo de Nelson-Siegel}

Nelson y Siegel desarrollaron un modelo de ajuste de estructuras temporales de tasas de interés que es lo suficientemente flexible como para representar las distintas formas que generalmente adoptan estas curvas: Monótonas, Jorobadas y en forma de $S$.

En el modelo paramétrico de Nelson-Siegel, se supone que la tasa forward instantánea es la solución de una ecuación diferencial de segundo orden con 
dos raíces iguales. Siguiendo la misma notación que en la sección anterior, suponiendo que la fecha de negociación de un contrato forward es $t=0$ y que el día de inicio de la operación subyacente es $m$, la función de la tasa forward instantánea se define según:

$$
f(m)=f(0, m)=\beta_{0}+\beta_{1} \exp \left(-\frac{m}{\tau}\right)+\beta_{2} \frac{m}{\tau} \exp \left(-\frac{m}{\tau}\right),
$$

donde $\left(\tau, \beta_{0}, \beta_{1}, \beta_{2}\right)$ es el vector de parámetros que determina la forma de la curva. Como se explicó en la sección anterior, la tasa spot $r(0, m)$ se obtiene integrando las tasas forward instantáneas desde 0 hasta $m$ y dividiendo entre $m$. De esta manera se obtiene la función de la tasa spot:

$$
r(m)=r(0, m)=\beta_{0}+\left(\beta_{1}+\beta_{2}\right)\left[1-\exp \left(-\frac{m}{\tau}\right)\right] / \frac{m}{\tau}-\beta_{2} \exp \left(-\frac{m}{\tau}\right) .
$$

Con el objeto de apreciar la flexibilidad que tiene este modelo para adaptarse a las formas que adoptan las curvas de estructura temporal de tasas de interés, a continuación se examinan los coeficientes de la función. Nótese que dichos coeficientes denotan el peso que se les da a las componentes de corto, mediano o largo plazo de la curva forward. En primer término, el límite de $r(m)$ cuando $m$ tiende a infinito es la constante $\beta_{0}$. Por lo tanto, el parámetro $\beta_{0}$ denota el peso que se le da a la componente de largo plazo. Análogamente, $\beta_{1}$ denota el peso que se le da a la componente de corto plazo y $\beta_{2}$ es la importancia relativa al inediano plazo en la estructura temporal de tasas.

Figura 1. Componentes de la curva forward.

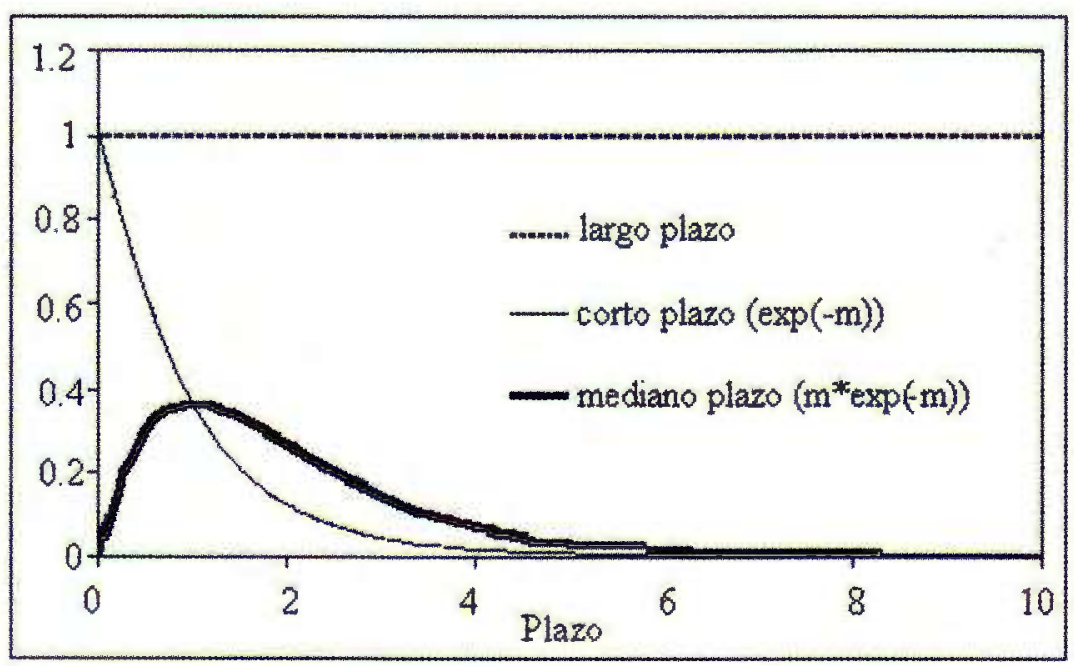

En la figura anterior, se muestran las distintas componentes de la curva forward. La componente de largo plazo es una constante que no cae a cero en el límite, 
mientras que la componente de mediano plazo está asociada aí único término que empieza en cero (por lo cual no es de corto plazo) y cae a cero (por lo cual no es de largo plazo). El término de corto plazo es el único que cae monótonamente a cero.

A su vez, el parámetro $\tau$ determina la rapidez con la que los términos que lo incluyen tienden a su límite. Entonces con un valor pequeño de $\tau$ las aproximaciones de la curva son mejores en los plazos cortos que en los largos. Análogamente, con valores de $\tau$ grandes, el ajuste es mejor en el largo plazo que en el corto. Esta situación se muestra en la siguiente figura. Para el ejemplo tratado, se observa que la curva ajustada con $\tau=90$ representa mejor la curvatura de los datos originales a corto plazo, a diferencia de la curva ajustada con $\tau=150$ que difiere más de la curva real en el corto plazo, pero se ajusta mejor a los datos de largo plazo. Nótese que de no ser por $\tau$, el modelo es lineal en los demás parámetros.

Figura 2. Datos y curvas ajustadas.

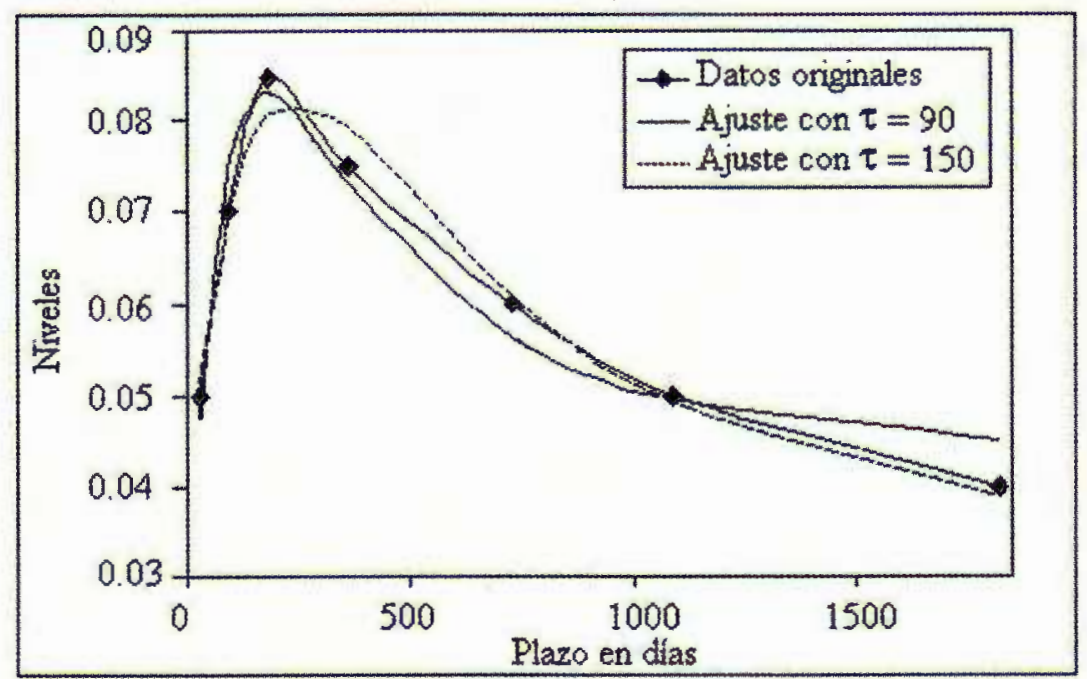

\section{Metodología para ajustar las estructuras temporales de tasas}

Como se describió en el apartado correspondiente, es necesario convertir las tasas que se encuentran en interés simple a distintos plazos observadas en un momento dado, a tasas continuamente compuestas. A estos datos originales se les ajusta una estructura de tasas de acuerdo al modelo de Nelson-Siegel.

Aunque se puede simplemente estimar los parámetros utilizando alguna paquetería convencional de mínimos cuadrados no lineales, se puede aprovechar con cierta eficiencia el hecho de que el nodelo es lineal en todos los parámetros menos en $\tau$. La forma empleada por los autores, es la de iterar sobre 
el parámetro que representa la no-linealidad del modelo, resolviendo sucesivamente regresiones lineales sobre los parámetros restantes hasta obtener convergencia bajo el criterio de maximizar la $R^{2}$. Así, se utiliza algún procedimiento de búsqueda unidimensional en la parte no-lineal del modelo, y se aprovecha la eficiencia de estimación en la parte lineal. Por facilidad, para la búsqueda unidimensional, escogimos el método de la "Sección Dorada". 5

Precisando, primero se reescribe la ecuación de Nelson-Siegel según:

$$
r(m)=a+b\left[1-\exp \left(-\frac{m}{\tau}\right)\right] / \frac{m}{\tau}+c \exp \left(-\frac{m}{\tau}\right),
$$

donde $r(m)$ representa la tasa continuamente compuesta al plazo $m$.

El proceso iterativo es el siguiente. Suponiendo que se tienen los datos de las tasas spot compuestas de manera continua a distintos plazos, $m_{1}, m_{2}, \ldots, m_{n}$, y para un cierto valor del parámetro $\tau$, los parámetros " $a$ ", " $b$ " y " $c$ ", se obtienen como la solución por mínimos cuadrados del sistema de ecuaciones, que en forma matricial se representa como:

$$
\begin{aligned}
& X=\left(\begin{array}{c}
r\left(m_{1}\right) \\
r\left(m_{2}\right) \\
\cdot \\
\cdot \\
\cdot \\
r\left(m_{n}\right)
\end{array}\right), M=\left(\begin{array}{ccc}
1 & {\left[1-\exp \left(-\frac{m_{1}}{\tau}\right)\right] / \frac{m_{1}}{\tau}} & \exp \left(-\frac{m_{1}}{\tau}\right) \\
1 & {\left[1-\exp \left(-\frac{m_{2}}{\tau}\right)\right] / \frac{m_{2}}{\tau}} & \exp \left(-\frac{m_{2}}{\tau}\right) \\
\cdot & \cdot & \cdot \\
\cdot & \cdot & \cdot \\
\cdot & {\left[1-\exp \left(-\frac{m_{n}}{\tau}\right)\right] / \frac{m_{n}}{\tau}} & \exp \left(-\frac{m_{n}}{\tau}\right)
\end{array}\right) \\
& \text { у } C=\left(\begin{array}{c}
a \\
b \\
c
\end{array}\right) .
\end{aligned}
$$

Entonces, el sistema de ecuaciones descrito por $X=M C$ es igual a:

$$
\left(\begin{array}{c}
r\left(m_{1}\right) \\
r\left(m_{2}\right) \\
\cdot \\
\cdot \\
\cdot \\
r\left(m_{n}\right)
\end{array}\right)=\left(\begin{array}{ccc}
1 & {\left[1-\exp \left(-\frac{m_{1}}{\tau}\right)\right] / \frac{m_{1}}{\tau}} & \exp \left(-\frac{m_{1}}{\tau}\right) \\
1 & {\left[1-\exp \left(-\frac{m_{2}}{\tau}\right)\right] / \frac{m_{2}}{\tau}} & \exp \left(-\frac{m_{2}}{\tau}\right) \\
\cdot & \cdot & \cdot \\
\cdot & \cdot & \cdot \\
\cdot & {\left[1-\exp \left(-\frac{m_{n}}{\tau}\right)\right] / \frac{m_{n}}{\tau}} & \exp \left(-\frac{m_{n}}{\tau}\right)
\end{array}\right)\left(\begin{array}{l}
a \\
b \\
c
\end{array}\right)
$$

Este sistema corresponde a un problema de regresión lineal múltiple y su solución está representada por las bien conocidas "ecuaciones normales", es decir: $C^{*}=\left(M^{T} M\right)^{-1} M^{T} X$. Una vez obtenido el vector $C^{*}$, calculamos el

5 En general, es más fácil hacer búsquedas unidimensionales que resolver el problema de golpe. Además esto conduce a la necesidad de escoger buenos puntos de inicio del proceso iterativo, que es más fácil con el método propuesto. 
vector de las tasas estimadas $\hat{X}$ en los mismos plazos de los datos originales con los valores obtenidos: $\hat{X}=M C^{*}$. En seguida se calcula la suma de los cuadrados de los errores y $R_{\text {ajustada }}^{2}$ correspondiente al modelo de Nelson-Siegel según:

$$
\begin{gathered}
\sum_{i=1}^{n} e_{i}^{2}=\left[(X-\hat{X})^{T}(X-\hat{X})\right], \\
R^{2}=1-\frac{\sum_{i=1}^{n} e_{i}^{2}}{\operatorname{Var}(X)}, \\
R_{\text {ajustada }}^{2}=1-\frac{n-1}{n-3}\left(1-R^{2}\right) .
\end{gathered}
$$

Hecho lo anterior, fijando los parámetros " $a$ ", " $b$ " y " $c$ ", se optimiza sobre $\tau$ mediante el método de la sección dorada dentro de un intervalo $[u, v]$. Con el nuevo valor del parámetro $\tau$ se repite el proceso hasta que se establece convergencia en $R^{2}$. De esta manera se obtiene el vector de parámetros $\left(\tau, \beta_{0}, \beta_{1}, \beta_{2}\right)$ que representa la estructura de tasas ajustada a los datos.

El método de la sección dorada para encontrar un máximo global, requiere que la función a optimizar sea unimodal y cuasicóncava ${ }^{6}$ De no ser así, solo se puede garantizar convergencia a un óptimo local. Cuando esto sucede, normalmente son dos los óptimos locales, los cuales están en los extremos del intervalo y la convergencia a uno u otro depende mucho del punto de inicio. Desgraciadamente, no se puede garantizar que la función a optimizar, que en nuestro caso es la $R^{2}$ ajustada a la función de Nelson-Siegel, sea unimodal y cuasicóncava con respecto al parámetro $\tau .^{7}$

Para los cuatro casos examinados en este artículo, las curvas de Cetes, Udibonos, Libor y T-Bills, se encontraron patrones de concavidad/convexidad mezclados. Sin embargo, no se encontró una forma general para elegir el intervalo de variabilidad de $\tau$ donde realizar la búsqueda. Por este motivo, se graficaron las funciones objetivo en algunos puntos equidistantes dentro de un intervalo amplio antes de realizar la optimización para elegir el dominio de la función.

Dependiendo de la forma de las curvas y de los plazos de los datos originales se elige un intervalo inicial donde se encuentra el valor óptimo de $\tau$. A continuación se muestran las gráficas del tipo de funciones encontradas en la investigación realizada.

6 Véase Márquez (1984).

7 Es necesario señalar, que el buen o mal comportamiento de $R^{2}$ como función de $\tau$, no tiene nada que ver con el algoritmo utilizado para optimizar. 
Figura 3. $R_{\text {ajustada }}^{2}$

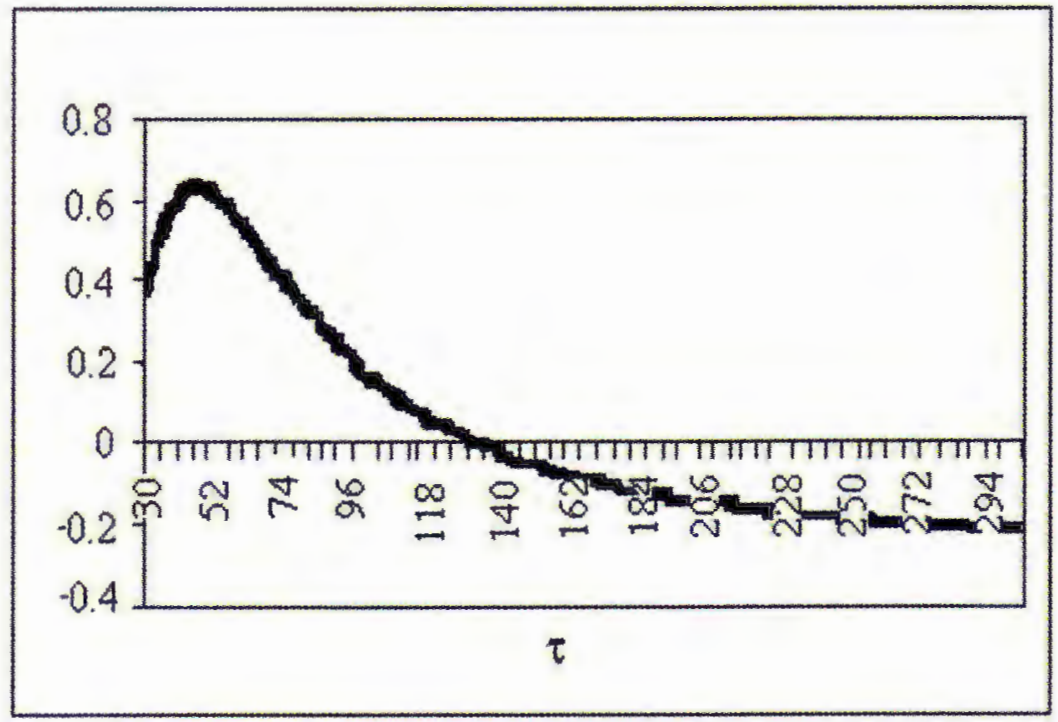

Figura 4. $R_{a j u s t a d a}^{2}$.

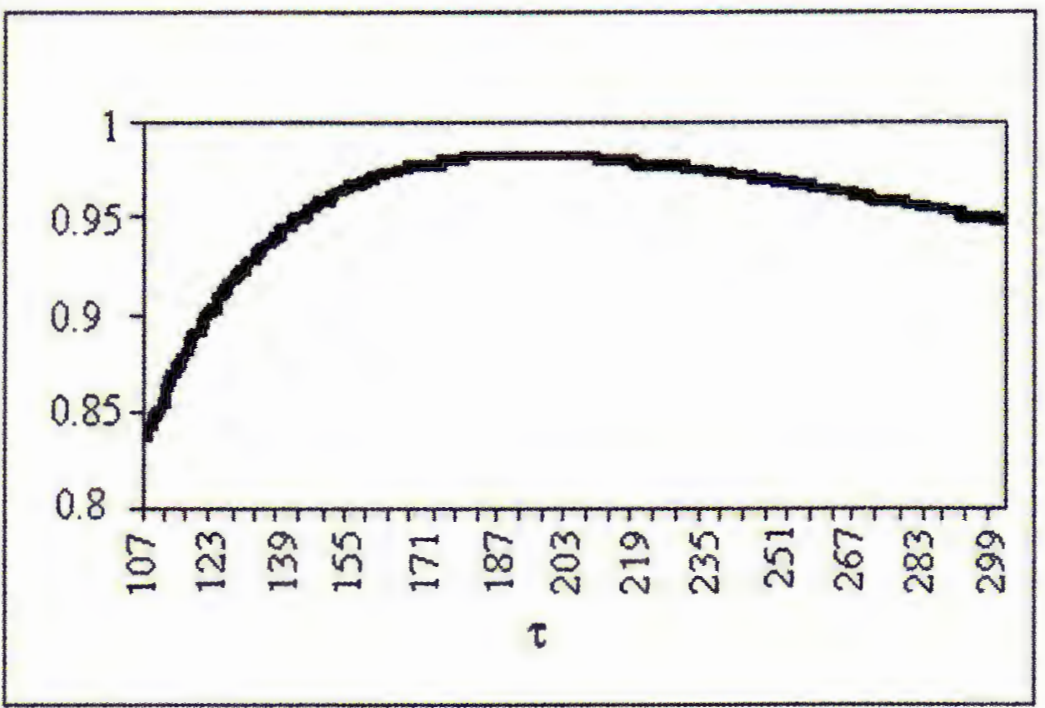


En las dos primeras figuras se tiene el caso en que la función de $R^{2}$ contra $\tau$ son unimodales y cuasicóncavas, además tienen su óptimo global en el interior del intervalo. Al ser bien comportadas, no se necesita tomar ninguna precaución adicional. En la siguiente figura, se aprecia una función que también es cuasicóncava, pero al ser monótona creciente en $\tau$, no es unimodal dentro del intervalo. Parecería que el óptimo está en $\tau=\infty$, lo cual no tiene sentido práctico.

Por lo tanto en este caso, se trunca el intervalo en el momento en que se tiene una buena precisión de la $R^{2}$ y se escoge el extremo correspondiente como el valor óptimo de $\tau$. No es recomendable alargar demasiado el intervalo, ya que el modelo estaría ajustando bien a largo plazo pero a expensas de un mal ajuste en el corto. Además, esto conduce a problemas de estabilidad numérica.

Figura 5. $R_{a j u s t a d a}^{2}$.

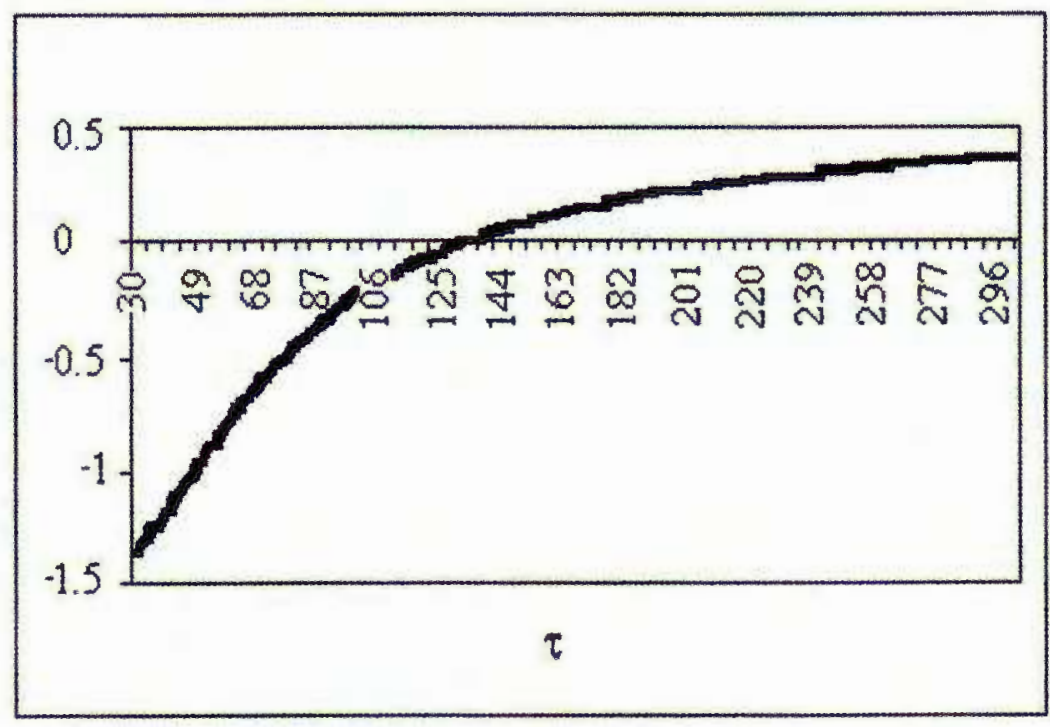

En la figura siguiente se muestra un caso en el que después del óptimo local alrededor de $\tau=50$, la función cae para luego volver a crecer indefinidamente, pero parece que nunca rebasará el máximo local mencionado. En este caso, acotamos el intervalo para que la búsqueda convergiera al óptimo local mencionado. 
Figura 6. $R_{\text {ajustada }}^{2}$

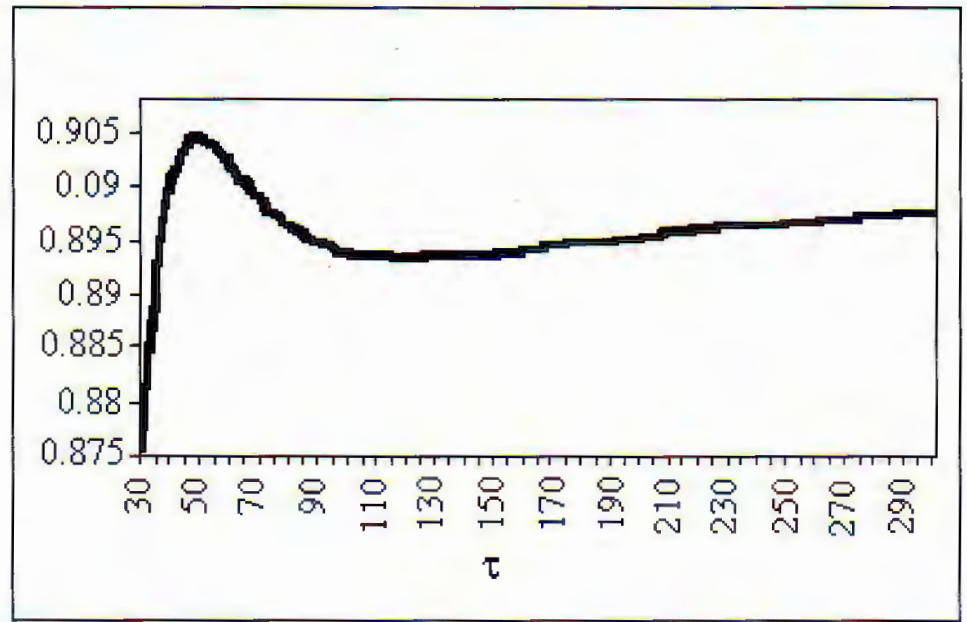

Finalmente, también se encontraron curvas que no eran cuasicóncavas y en donde los óptimos locales estaban en los dos extremos del intervalo. En este caso se escogió el mayor de los dos; el de la izquierda, en el caso mostrado en la Figura 7.

Figura 7. $R_{a j u s t a d a}^{2}$

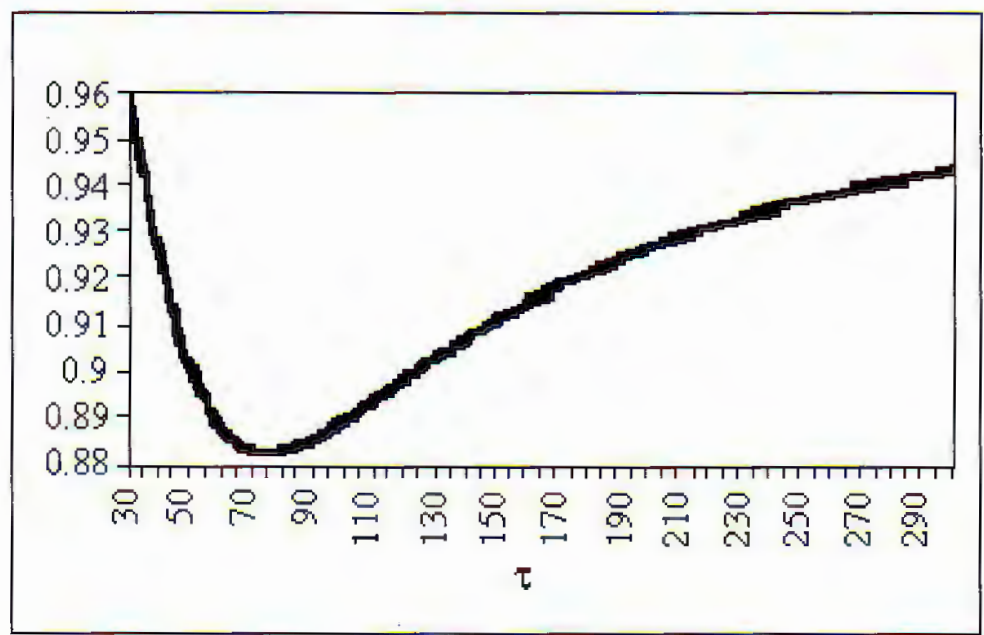


Por lo anterior, cada caso debe analizarse con cuidado para obtener un buen ajuste de los parámetros. Por ejemplo, para la curva de Cetes, inicialmente se corrió el algoritmo de la sección dorada dejando que $\tau$ variara en el intervalo $(1,1000)$, pero resultaban $\beta_{0}$ negativas. Es decir, a largo plazo las tasas estimadas tomarían valores negativos, mientras que a plazos cortos las tasas eran exageradamente altas. Después de varias pruebas se resolvió el problema acotando el intervalo a $(10,364)$, ya que 364 es el plazo mayor para el que se tienen datos históricos. Finalmente, se tomó la decisión de correr el modelo hasta el plazo mayor para el que se tuvieran datos de cada uno de los instrumentos restantes.

\section{Simulación de estructuras temporales}

Para propósitos de evaluación de la técnica e ilustración, se presentan los resultados de los ajustes a las curvas de estructura temporal de tasas para Cetes, Udibonos, Libor y T-Bills. Se construyeron las cuatro series de los parámetros correspondientes a partir de datos diarios desde enero de 2001 hasta enero de 2002. Para cada uno de los parámetros $\left(\left(\tau, \beta_{0}, \beta_{1}, \beta_{2}\right)\right.$ en ese orden), se obtuvo su histograma de frecuencias y se calculó su matriz de varianzas-covarianzas. Una vez realizada esta operación, se aplicó la factorización de Cholesky " $A$ ", de tal manera que $\Delta=A A^{\prime}$. Los histogramas de los parámetros se toman como distribuciones de probabilidad empíricas para estos. El esquema de simulación es simplemente el de aplicar la fórmula siguiente:

$$
\pi=\left(\begin{array}{c}
\tau \\
\beta_{0} \\
\beta_{1} \\
\beta_{2}
\end{array}\right)=\mu+A \theta .
$$

Donde $\pi$ denota el vector de parámetros simulado, $\mu$ es el vector de valores medios de los parámetros, $A$ es la factorización de Cholesky de la matriz de varianza-covarianza de los parámetros y $\theta$ es un vector de muestras aleatorias de las correspondientes distribuciones empíricas de los parámetros. ${ }^{8}$ Cabe señalar que para poder utilizar este proceso de simulación se tuvo que estandarizar la distribución empírica de cada uno de los parámetros, restándole a cada intervalo su media y dividiéndola entre la desviación estándar de la distribución, para que no se duplicaran los efectos de multiplicar el vector por la matriz, con la factorización de Cholesky. Por medio de este proceso se pueden generar tantas curvas de estructuras temporales como se desee.

\subsection{Ejemplos del ajuste de curvas y distribuciones de parámetros}

A continuación se muestra el ajuste de la estructura temporal, el día 28 de enero de 2002 para los datos de Cetes. En los cuadros se observan los datos iniciales sobre los cuales se ajustó el modelo de Nelson-Siegel y los valores que toma la curva ajustada en algunos de los plazos. Se grafican los puntos

8 Esto se logra generando números aleatorios uniformemente distribuídos entre cero y uno (i.e. $\xi \approx U[0,1]$ ) y asociándolos con el intervalo correspondiente de la distribución acumulada empírica de cada uno de los parámetros, a la manera de Monte Carlo. 
originales y la curva que se forma al sustituir el valor óptimo de los parámetros. Posteriormente, se presentan las proporciones observadas de las curvas generadas por las simulaciones y de las curvas originales para las que se ajustó el modelo, agrupándolas de acuerdo a las formas que se observan más comúnmente en la realidad; a saber: normales, invertidas y mezcladas.

Figura 8. Tipo de estructuras de tasas.

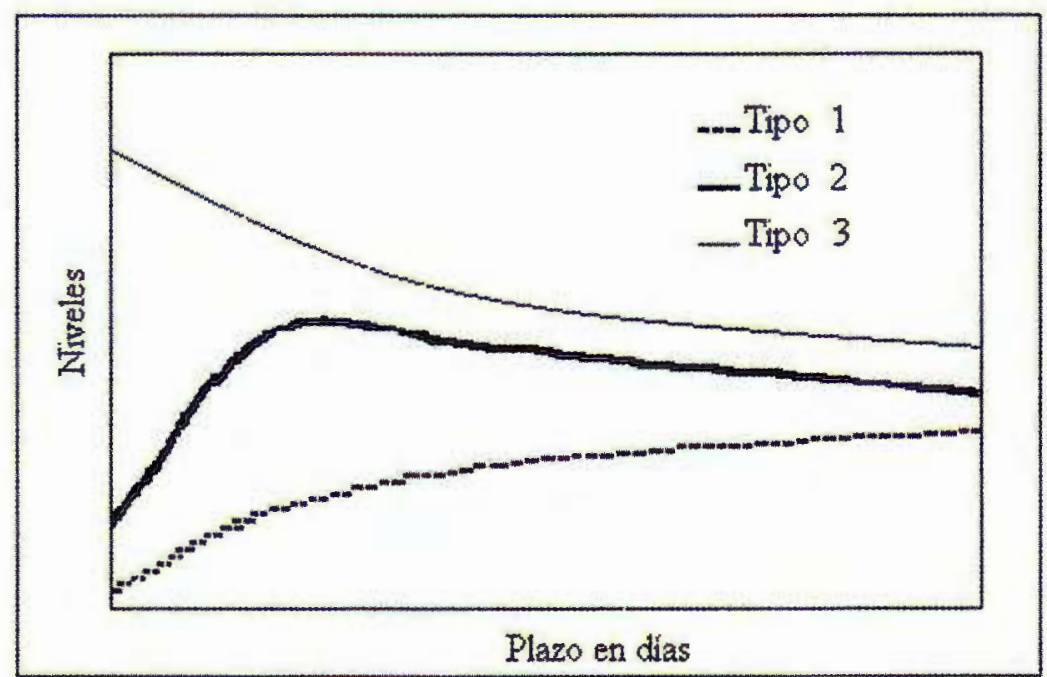

La estructura de Tipo 1 , se conoce como una estructura "normal", donde la tasa es una función creciente del plazo, es decir, a mayor plazo mayor tasa ya que la incertidumbre crece con el tiempo. La estructura de Tipo 2 se conoce como una estructura "mezclada"; es creciente en el plazo hasta cierto punto y después se hace decreciente en el largo plazo. El último tipo de curva se conoce como una estructura "invertida", ya que la tasa es decreciente para todo plazo, indicando que las tasas de interés de corto plazo son muy altas y que la expectativa de los inversionistas es de que bajen en el futuro para regresar a niveles mas "normales". Posteriormente se mostraran sólo los resultados más importantes que se obtuvieron para los demás instrumentos para los que se realizó el ajuste de las estructuras temporales de tasas, además de indicar el proceso que se siguió para realizar el ajuste y los resultados obtenidos por la simulación Monte Carlo. 


\subsection{Ejemplo: Cetes}

\subsubsection{Ajuste de las curvas}

Para la curva de Cetes se decidió trabajar con los plazos generalmente observados en el mercado, que por lo general corresponden a 28, 91, 182 y 364 días. En la primera tabla, se muestran los datos de las tasas de Cetes al cierre de operaciones del 28 de enero del $2002 .^{9}$ El vector de parámetros óptimo para la curva de Cetes a esta fecha, la cual se obtuvo mediante la técnica descrita en el apartado cuatro, es el siguiente:

$$
\left(\tau, \beta_{0}, \beta_{1}, \beta_{2}\right)=\left(\begin{array}{llll}
64.58968 & 0.09857 & -0.01706 & -0.03324
\end{array}\right)
$$

Tabla 1.a

\begin{tabular}{|c|c|c|}
\hline \multicolumn{3}{|c|}{ Datos Cetes para el 28/01/2002 } \\
\hline Plazo & Tasa Simple & Tasa Continua \\
\hline 28 & 0.07222 & 0.07202 \\
\hline 91 & 0.07679 & 0.07605 \\
\hline 182 & 0.08250 & 0.08083 \\
\hline 364 & 0.09176 & 0.08775 \\
\hline
\end{tabular}

Tabla 1.b

\begin{tabular}{|c|c|c|}
\hline \multicolumn{3}{|c|}{ Ajuste Cetes } \\
\hline Plazo & Tasa Simple & Tasa Continua \\
\hline 7 & 0.07057 & 0.07052 \\
\hline 28 & 0.07221 & 0.07201 \\
\hline 91 & 0.07677 & 0.07604 \\
\hline 182 & 0.08250 & 0.08083 \\
\hline 364 & 0.09176 & 0.08775 \\
\hline
\end{tabular}

En la tabla 1.b y la Figura 9, se muestran numérica y gráficamente el ajuste obtenido a partir del modelo propuesto por Nelson-Siegel.

9 Los datos se tomaron del archivo de curvas de Valmer, el proveedor de precios. En este archivo se muestran tanto las tasas observadas en el mercado, como las tasas estimadas por el proveedor. Es importante notar que las estimaciones del proveedor para Cetes de largo plazo son muy similares en todos los días del año. 
Figura 9. Tasa simple Cetes.

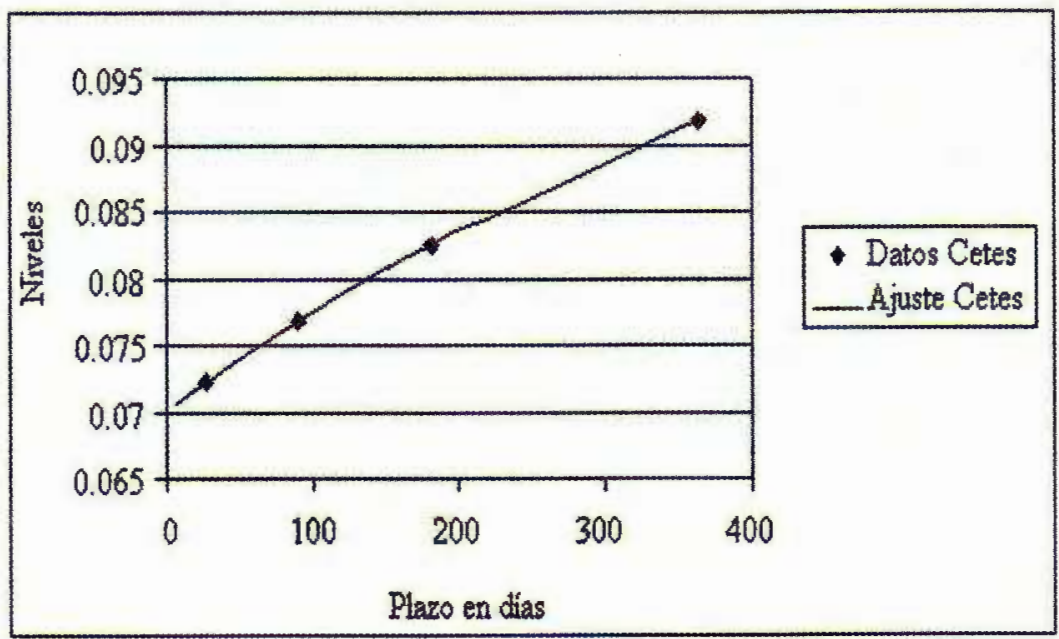

\subsubsection{La distribución empírica de los parámetros}

A partir de la serie de los cuatro parámetros $\left(\tau, \beta_{0}, \beta_{1}\right.$ y $\left.\beta_{2}\right)$ se obtuvo el vector de medias y la matriz de varianzas-covarianzas, $\Delta$. Para el caso de los Cetes, los resultados obtenidos son:

$$
\begin{gathered}
\left(\bar{\tau}, \overline{\beta_{0}}, \overline{\beta_{1}}, \overline{\beta_{2}}\right)=\left(\begin{array}{llll}
75.05079 & 0.11922 & 0.03342 & -0.07385
\end{array}\right), \\
\Delta=\left(\begin{array}{cccc}
4647.543 & 1.13685 & 9.69502 & 8.56692 \\
1.13685 & 0.00038 & 0.00219 & 0.00376 \\
9.69502 & 0.00219 & 0.02565 & 0.01409 \\
8.56692 & 0.00376 & 0.01409 & 0.05595
\end{array}\right) .
\end{gathered}
$$

La matriz $A$ que resulta después de aplicar la factorización de Cholesky a $\Delta$ es la siguiente:

$$
A=\left(\begin{array}{cccc}
68.17289 & 0 & 0 & 0 \\
0.01667 & 0.01022 & 0 & 0 \\
0.14221 & -0.01771 & 0.07149 & 0 \\
0.12566 & 0.16336 & -0.01247 & 0.11538
\end{array}\right)
$$


A continuación se presentan los histogramas correspondientes a cada uno de los parámetro normalizados. Recordemos que estas figuras muestran los histogramas obtenidos para los parámetros de las curvas de tasas de Cetes.

Figura 10. Parámetro $\tau$.

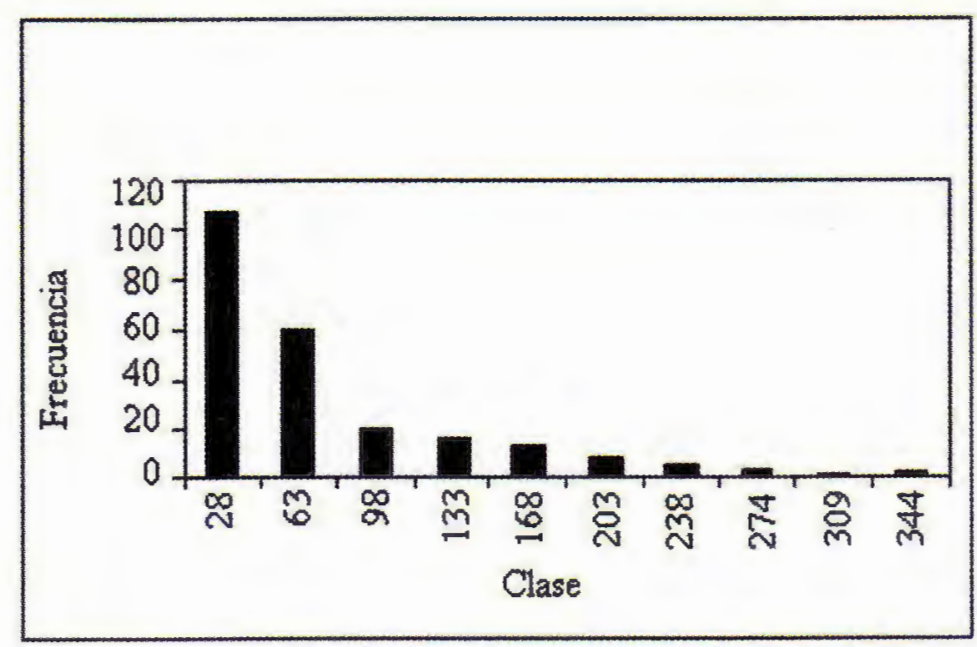

Figura 11. Parámetro $\beta_{0}$.

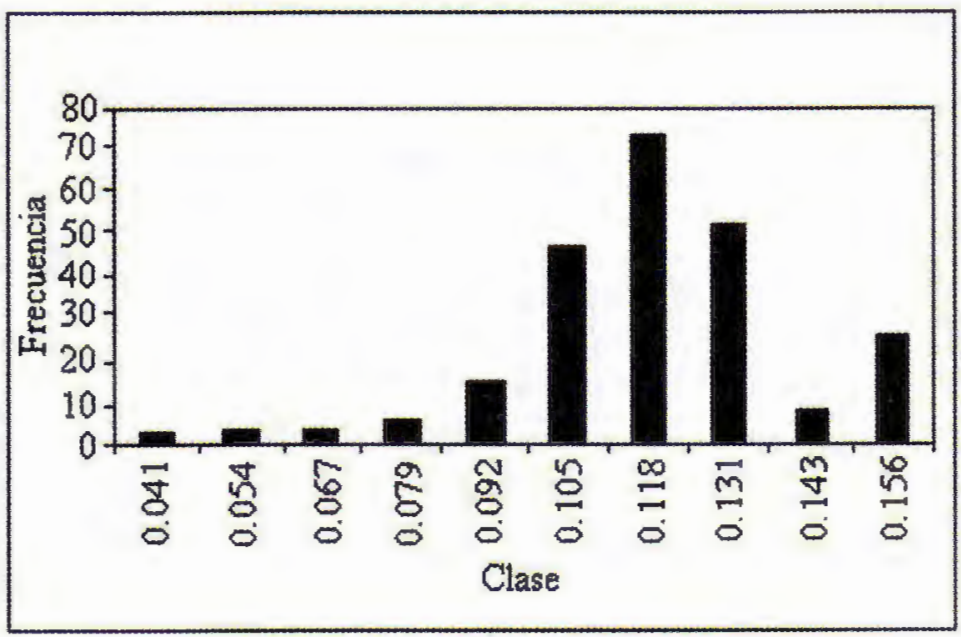


Figura 12. Parámetro $\beta_{1}$.

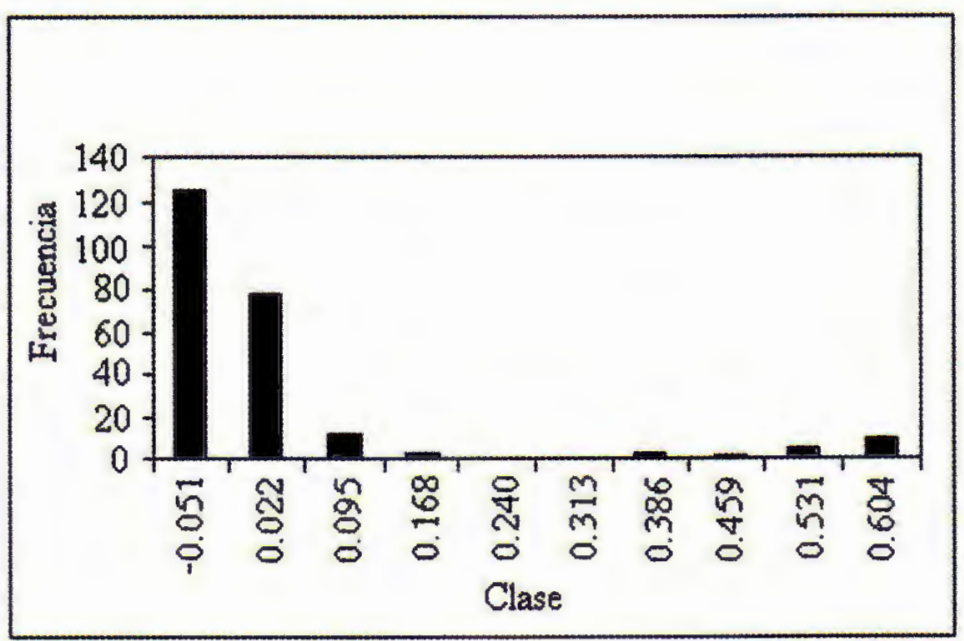

Figura 13. Parámetro $\beta_{2}$.

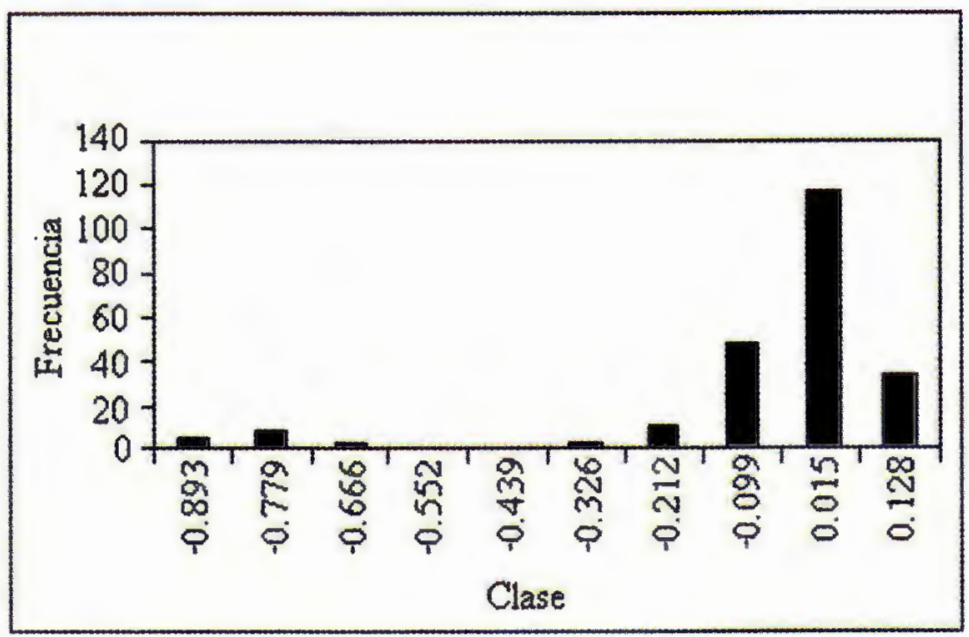


En las siguientes figuras se presenta las proporciones de las estructuras generadas por la simulación, así como las estructuras originales a las que se ajustó el modelo, según el tipo de estructura de tasas. Se puede observar que ambas gráficas son muy parecidas a la estructura de Tipo 1. Finalmente, aparece una figura que muestra algunas de las estructuras generadas por la simulación.

Figura 14. Forma de las curvas originales.

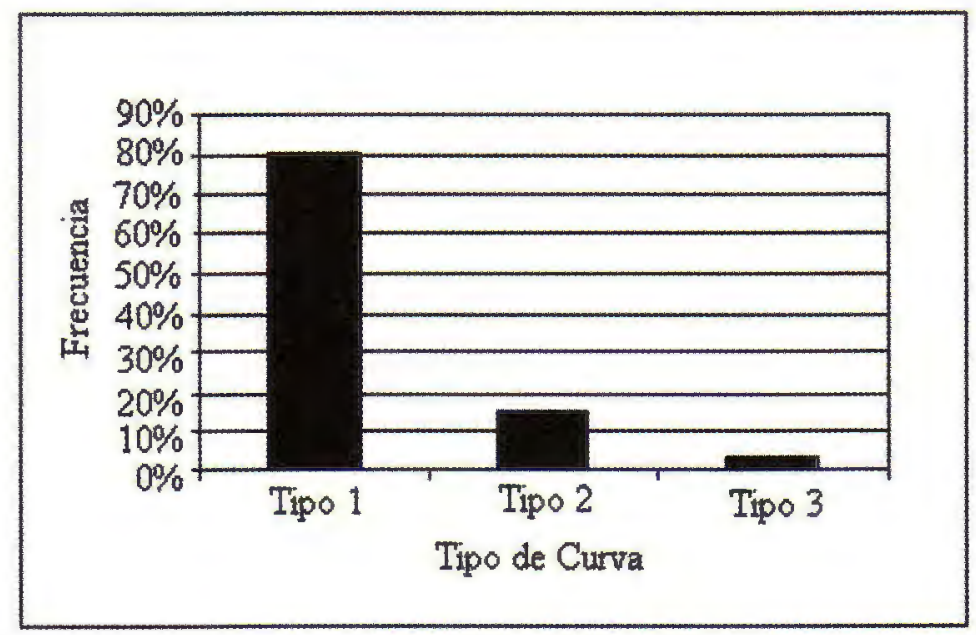

Figura 15. Forma de las curvas generadas por la simulación.

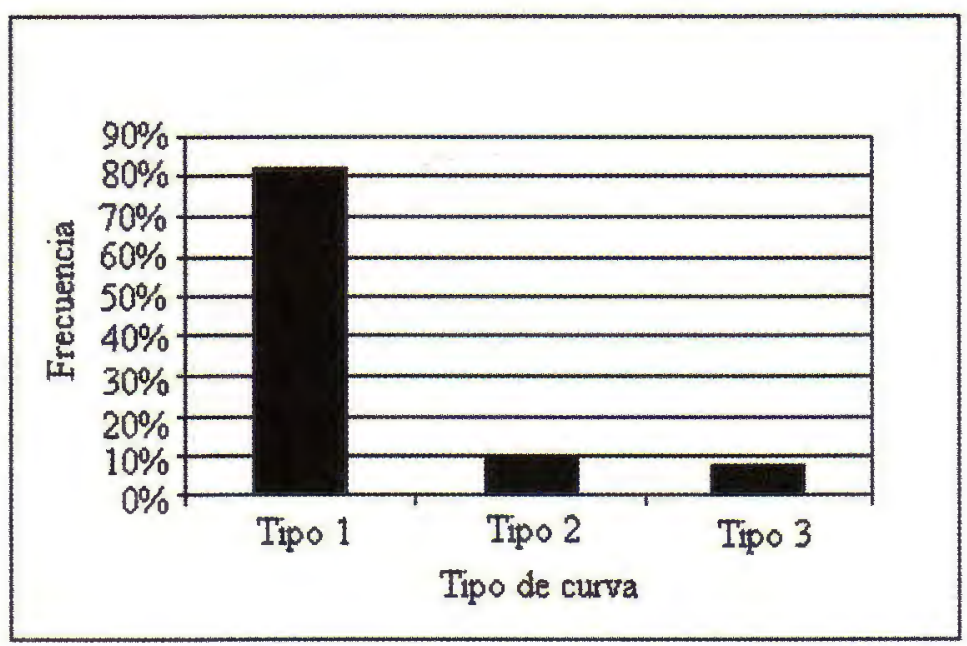


Figura 16. Estructuras generadas por la simulación para Cetes.

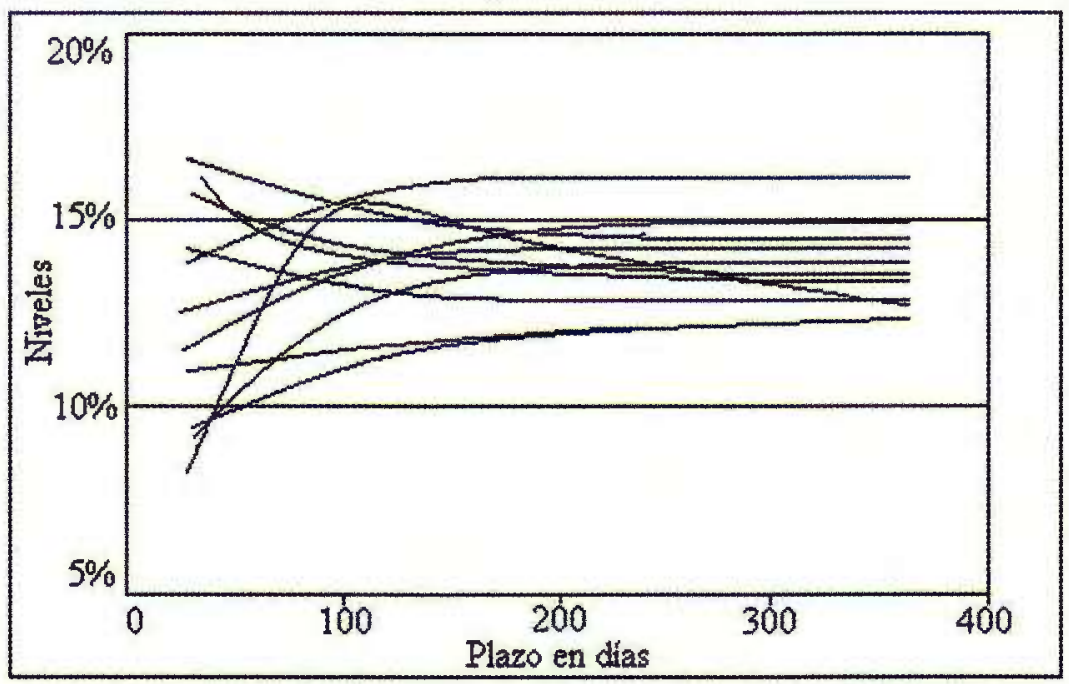

\subsection{Ejemplos: Udibonos, Libor y T-Bill}

\subsubsection{Resumen}

A continuación se presenta una tabla que resume los vectores de medias, las matrices de varianzas-covarianzas y la de Cholesky provenientes de los parámetros estimados, los cuáles se emplearon para sus respectivas simulaciones de estructuras. Los resultados completos sobre los ajustes, los histogramas y el proceso de simulación se presentan en los anexos dos, tres y cuatro.

Tabla 2.

\begin{tabular}{|c|c|c|c|c|}
\hline & \multicolumn{4}{|c|}{ Cetes } \\
\hline Media & $\begin{array}{c}\tau \\
75.05\end{array}$ & $\begin{array}{c}\beta_{0} \\
0.119223 \quad 0\end{array}$ & $\begin{array}{cc}\beta_{1} & \\
0.033422 & -0\end{array}$ & $\begin{array}{l}\beta_{2} \\
073859\end{array}$ \\
\hline \multicolumn{5}{|c|}{ Varianza-Covarianza } \\
\hline$\tau$ & 4647.54 & -0.434628 & $8-2.710816$ & 7.344585 \\
\hline$\beta_{0}$ & -0.434628 & 0.000382 & -0.000559 & 0.000675 \\
\hline$\beta_{1}$ & -2.710816 & -0.000559 & 0.025648 & -0.03486 \\
\hline$\beta_{2}$ & 7.344585 & 0.000675 & -0.034867 & 0.055948 \\
\hline \multicolumn{5}{|l|}{ Cholesky } \\
\hline $\bar{T}$ & 68.17 & 0 & 0 & 0 \\
\hline$\beta_{0}$ & -0.006375 & 0.018488 & 0 & 0 \\
\hline$\beta_{1}$ & -0.039764 & -0.043935 & 0.148784 & 0 \\
\hline$\beta_{2}$ & 0.107735 & 0.073639 & -0.183809 & 0.071641 \\
\hline
\end{tabular}


Tabla 2. (Continuación).

\begin{tabular}{|c|cccc|}
\hline & \multicolumn{4}{|c|}{ Libor } \\
\hline Media & $\tau$ & $\beta_{0}$ & $\beta_{1}$ & $\beta_{2}$ \\
& 45.30 & 0.036756 & 0.001787 & -0.004639 \\
\hline Varianza-Covarianza & \multicolumn{5}{|c}{} \\
\hline$\tau$ & 204.60 & -0.004286 & -0.015883 & -0.03575 \\
$\beta_{0}$ & -0.004286 & 0.000090 & 0.000031 & 0.000047 \\
$\beta_{1}$ & -0.015883 & 0.000031 & 0.000025 & 0.000028 \\
$\beta_{2}$ & -0.035756 & 0.000047 & 0.000028 & 0.000054 \\
\hline Cholesky & \multicolumn{5}{|c}{0} \\
\hline$\tau$ & 14.30 & 0 & 0 & 0 \\
$\beta_{0}$ & -0.000300 & 0.009476 & 0 & 0 \\
$\beta_{1}$ & -0.001110 & 0.003243 & 0.003618 & 0 \\
$\beta_{2}$ & -0.002500 & 0.004847 & 0.002535 & 0.004214 \\
\hline
\end{tabular}

\begin{tabular}{|c|cccc|}
\hline & \multicolumn{4}{|c|}{ Udibonos } \\
\hline \multirow{2}{*}{ Media } & $\tau$ & $\beta_{0}$ & $\beta_{1}$ & $\beta_{2}$ \\
& 534.30 & 0.041488 & 0.002152 & 0.060604 \\
\hline Varianza-Covarianza & \multicolumn{4}{c}{} \\
\hline$\tau$ & 356170.82 & -0.46327 & 10.45308 & -17.9785 \\
$\beta_{0}$ & -0.463274 & 0.000037 & -0.00008 & 0.000003 \\
$\beta_{1}$ & 10.453084 & -0.000081 & 0.00094 & -0.00089 \\
$\beta_{2}$ & -17.978599 & 0.000003 & -0.00089 & 0.001323 \\
\hline Cholesky & & & & \\
\hline$\tau$ & 596.80 & 0 & 0 & 0 \\
$\beta_{0}$ & -0.000776 & 0.006074 & 0 & 0 \\
$\beta_{1}$ & 0.017515 & -0.011072 & 0.022794 & 0 \\
$\beta_{2}$ & -0.030125 & -0.003368 & -0.017837 & 0.009258 \\
\hline
\end{tabular}

\begin{tabular}{|c|c|c|c|c|}
\hline & \multicolumn{4}{|c|}{ T-Bill } \\
\hline Media & $\begin{array}{c}\tau \\
2862.87\end{array}$ & $\begin{array}{cc} & \beta_{0} \\
7 & 0.01941\end{array}$ & $\begin{array}{c}\beta_{1} \\
0.01178 \quad 0\end{array}$ & $\begin{array}{c}\beta_{2} \\
0.04736\end{array}$ \\
\hline \multicolumn{5}{|l|}{ Varianza-Covarianza } \\
\hline $\bar{\tau}$ & 2557635.2 & -2.3787 & 12.3078 & -27.0345 \\
\hline$\beta_{0}$ & -2.3787 & 0.000028 & -0.000016 & -0.000055 \\
\hline$\beta_{1}$ & 12.3078 & -0.000016 & 0.000152 & -0.000244 \\
\hline$\beta_{2}$ & -27.0345 & -0.000055 & -0.00024 & 0.000743 \\
\hline \multicolumn{5}{|l|}{ Cholesky } \\
\hline$\tau$ & 1599.26 & $\overline{0}$ & 0 & 0 \\
\hline$\beta_{0}$ & -0.001487 & 0.005098 & 0 & 0 \\
\hline$\beta_{1}$ & 0.007696 & -0.000940 & 0.009609 & 0 \\
\hline$\beta_{2}$ & -0.016904 & -0.015742 & -0.013433 & $3 \quad 0.005354$ \\
\hline
\end{tabular}




\subsubsection{Obtención de los datos y estimación de los parámetros}

Los datos para Udibonos se tomaron del archivo "vector" de Valmer. En este archivo se muestran tanto el rendimiento de los Udibonos observado en el mercado como el rendimiento estimado por el proveedor de precios. A diferencia del archivo de Valmer del cual se obtuvieron los datos para los Cetes, en los Udibonos no se sabe cuáles datos corresponden a transacciones hechas en el mercado y cuáles son estimados por Valmer. Por esta razón, se trabajó con todos los plazos publicados.

Los plazos de los datos publicados diariamente varían entre 10 y 3700 días. Se corrió el algoritmo de optimización dejando que $\tau$ variara en el intervalo $(10,3700)$. Durante los primeros 6 meses del año 2001, se publicaron rendimientos de Udibonos para plazos de 15 días en adelante. En el segundo semestre, los plazos publicados empiezan a partir de los 200 días aproximadamente. Es decir, el modelo se ajusta con datos a partir de 200 días y se usa para pronosticar tasas de corto plazo.

En tanto que para los datos de la tasa Libor, se usó la base de datos de Bloomberg. Las tasas publicadas corresponden a los plazos 7, 28, 91, 182, 273 y 365 días. Si se corría el algoritmo dejando que $\tau$ variara en un intervalo muy grande, resultaban $\beta_{0}$ negativas. Esto generaba valores negativos para las tasas estimadas de largo plazo, mientras que las tasas de corto plazo tomaban valores muy altos respecto a los observados. Después de varias pruebas y de acuerdo con los resultados observados, el problema se resolvió acortando el intervalo a $(10,150)$. Como consecuencia, el valor óptimo de $\tau$ era en general, menor a 100.

Por último, para los datos de las tasas del T-Bill tambien se empleó a Bloomberg. Es importante notar, que en general se tienen los datos a 91, $182,365,730,1825,3650$ y 10950 días. Hay tasas de muy largo plazo y la $\tau$ óptima es alta (la media es 2857). Esto significa que la curva se ajusta mejor en el largo plazo que en el corto. Entonces, si se desea ajustar el corto plazo debería reducirse el intervalo del parámetro $\tau$, o bien, hacer la optimización de parámetros ignorando los datos de los plazos largos.

Al principio se ajustó el modelo dejando que $\tau$ variara en el intervalo $(1,1000)$. El resultado fue que el parámetro tomó siempre su valor extremo en 999, es decir, el intervalo era demasiado corto. Al tomar un intervalo mayor, $(500,6000)$, en general el parámetro $\tau$ encontraba valores óptimos en el interior del intervalo.

\subsubsection{Resultados obtenidos}

Como los histogramas de los parámetros ajustados en los primeros casos resultaron en figuras muy regulares, se pensó en ajustar alguna distribución conocida a los parámetros estimados, simplificando aún más el proceso de Simulación Monte Carlo. Sin embargo, este no es el caso general, ya que al revisar los histogramas de los parámetros para instrumentos como Udibonos y Libor (mostrados en las figuras siguientes) no se observó el mismo comportamiento suave del histograma. Por lo tanto, se optó por simular parámetros directamente de su distribución empírica. 
Figura 17. Histograma del parámetro $\beta_{2}$ Udibinos.

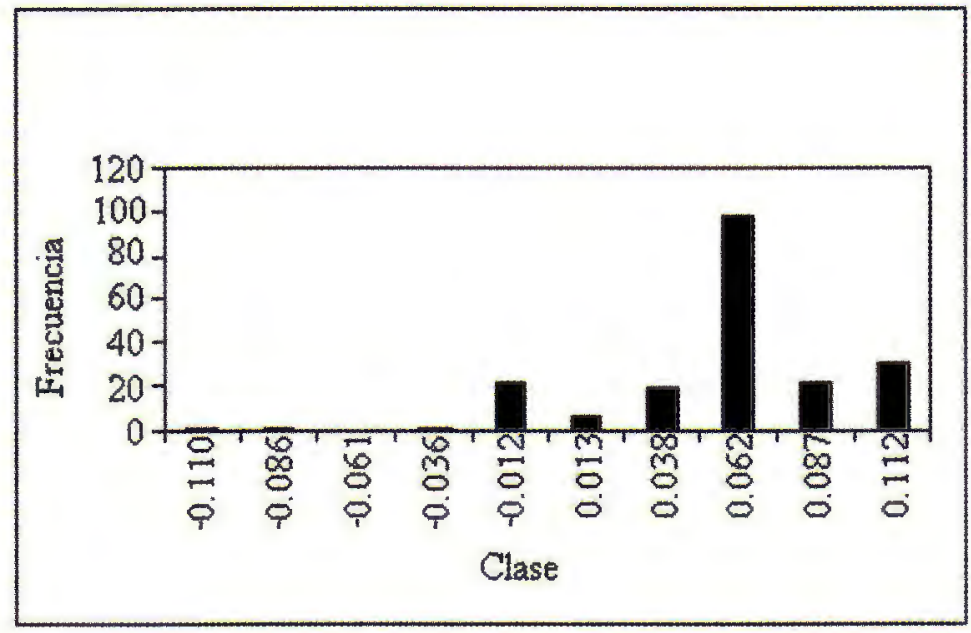

Figura 18. Histograma del parámetro $\beta_{0}$ Libor.

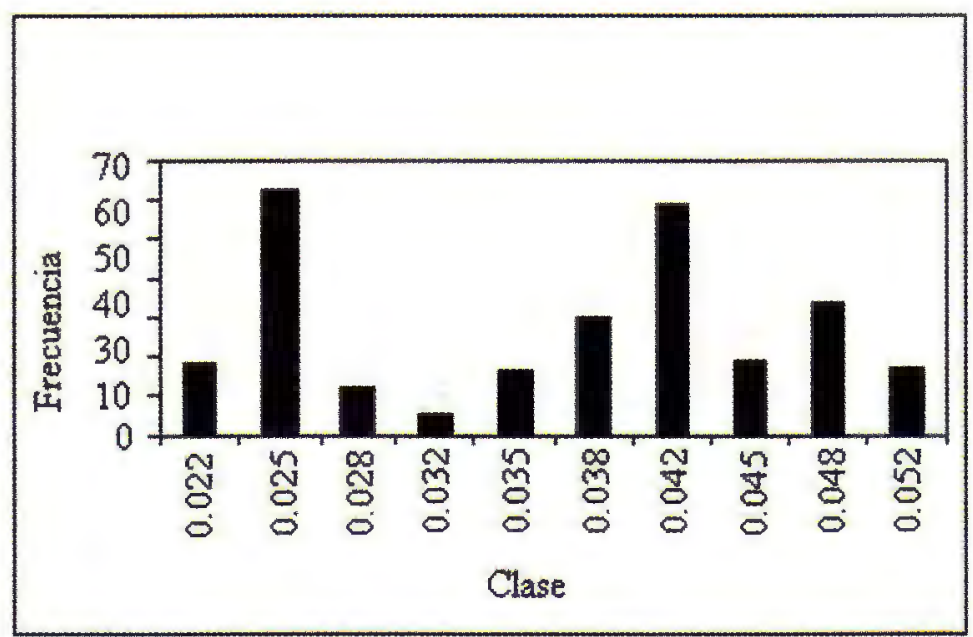


Figura 19. Histograma del parámetro $\beta_{1}$ T-Bill.

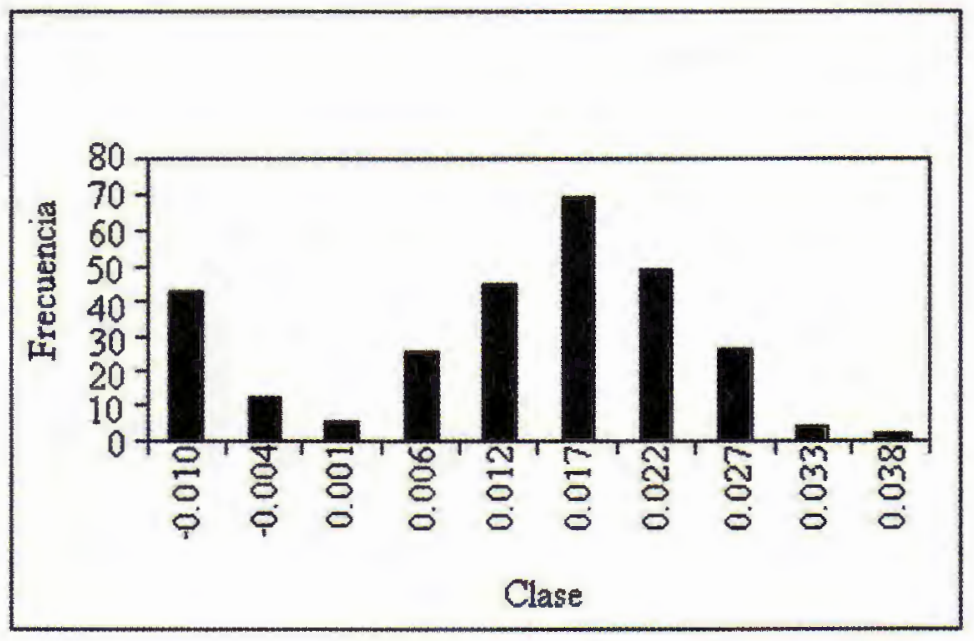

Figura 20. Histograma del parámetro $\beta_{2}$ T-Bill.

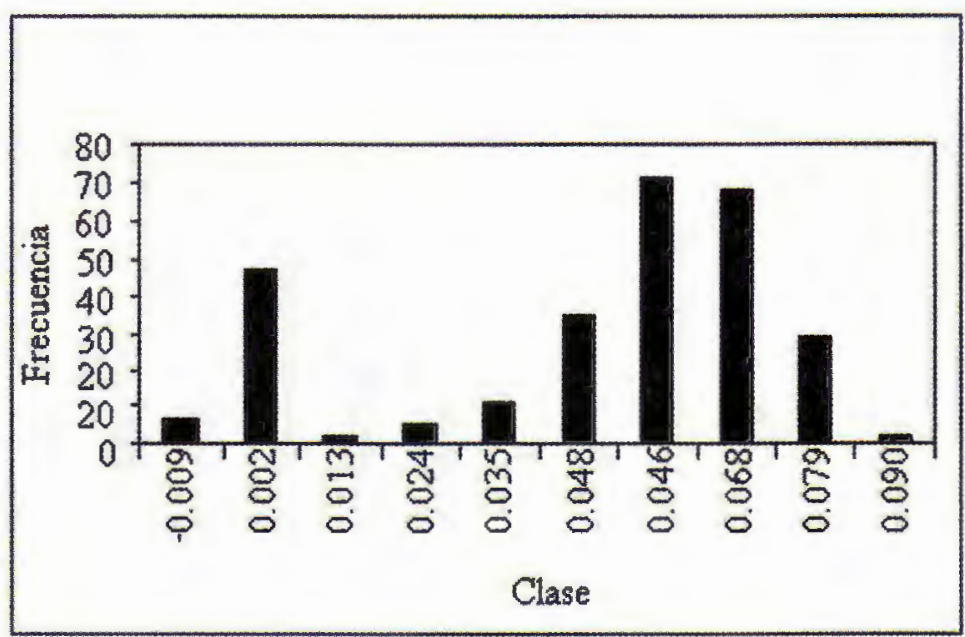

También se puede observar que los parámetros más problemáticos fueron $\beta_{0}, \beta_{1}$ y $\beta_{2}$. Es decir, los parámetros asociados a las componentes de corto, mediano y largo plazo de la curva forward. 


\section{Conclusiones}

Como suele suceder en este tipo de trabajos empíricos, se presentaron ciertas dificultades para la estimación de los parámetros, las cuales se resolvieron examinando los datos en cuestión y haciendo una utilización juiciosa del algoritmo de optimización de mínimos cuadrados no lineales por parte del analista.

Con el objeto de validar la metodología de simulación de curvas de tasas, en este trabajo solo fue necesario simular 2000 estructuras temporales para obtener convergencia en los parámetros de las distribuciones. Para efectos del cálculo del VaR de una cartera de instrumentos financieros de deuda, mediante la simulación Monte Carlo, idealmente se deben simular tantas estructuras como se requieran para garantizar convergencia en los parámetros, con algún nivel de precisión adecuado. Hasta la fecha, con carteras típicas de bancos mexicanos, esto puede significar simular un número de curvas en exceso de 10,000. De aquí el interés en el modelo de Nelson-Siegel, ya que implica ahorros significativos de tiempo y esfuerzo, al quedar completamente especificada la curva con cuatro parámetros.

Quizás el mayor defecto del modelo de Nelson-Siegel es que el ajuste puede dejar algo que desear en ciertos plazos, sobre todo en curvas que contemplan plazos muy largos. Es decir, dependiendo del valor del parámetro $\tau$ la curva se ajusta bien en el corto plazo o en el largo, pero no en ambos. Esta situación se ilustra en la tercera sección de este trabajo. Una conjetura interesante es la de incorporar un nuevo término a la ecuación que pueda arreglar el problema. Además, posiblemente se pueda lograr que este término le de mejores propiedades de concavidad a la función, con el fin de facilitar la optimización, pagando un precio pequeño aumentando solamente un parámetro.

Finalmente, otro asunto que queda pendiente es el de encontrar correlaciones entre los parámetros de las curvas asociadas a diferentes instrumentos. Esto redondearía el proceso, ya que aunque aumente considerablemente la dimensión del proceso de simulación, permitiría simular conjuntos de curvas, todas congruentes entre sí, para lograr una mejor representación de escenarios de tasas de interés. Pero esto se reduce simplemente a obtener la matriz de covarianzas entre todos los parámetros y queda igual el esquema de simulación.

\section{Apéndice 1. Método de la Sección Dorada}

El método de la Sección Dorada es un método iterativo en el cual se va acortando el intervalo de búsqueda del óptimo de una función univariada. Para el caso en el que se desea maximizar la $R^{2}$ y ajustar la ecuación de Nelson-Siegel sobre $\tau$, se hizo la búsqueda hasta obtener un intervalo de longitud menor o igual a 1. A continuación se explica el algoritmo de optimización para una minimización, tomando $[10, m]$ como el intervalo de inicio, donde $m$ representa el plazo mayor para el que se pudieron obtener datos de cada una de las estructuras temporales de las tasas de interés.

Sea $\left[u_{1}, v_{1}\right]$ el intervalo inicial sobre el cual se hará la búsqueda del punto óptimo. Los subíndices indican el número de iteración en el que se encuentra lá búsqueda. Denotemos por $k$ el contador de iteraciones. Así que en esta primera iteración $k=1$. Sean $\lambda_{1}=u_{1}+(1-\alpha)\left(v_{1}-u_{1}\right)$ y $\mu_{1}=u_{1}+\alpha\left(v_{1}-u_{1}\right)$ donde $\alpha=0.618$. Se calculan las regresiones para $\tau=\lambda_{1}, \tau=\mu_{1}$ y se repiten los siguientes pasos: 
1) Si $v_{k}-u_{k}<1$ detenerse, la solución óptima está en el intervalo $\left[u_{k}, v_{k}\right]$. De otro modo, si $R^{2}\left(\mu_{k}\right)>R^{2}\left(\lambda_{k}\right)$, ir al paso 2 y si $R^{2}\left(\mu_{k}\right) \leq R^{2}\left(\lambda_{k}\right)$, ir al paso 3.

2) $u_{k+1}=\lambda_{k}$ y $v_{k+1}=v_{k}$. Además sean $\lambda_{k+1}=\mu_{k}$ y $\mu_{k+1}=u_{k+1}+\alpha\left(v_{k+1}-\right.$ $\left.u_{k+1}\right)$. Se calcula $R^{2}\left(\mu_{k+1}\right)$ e ir al paso 4 .

3) $u_{k+1}=u_{k}$ y $v_{k+1}=\mu_{k}$. Además sean $\mu_{k+1}=\lambda_{k}$ y $\lambda_{k+1}=u_{k+1}+(1-$ $\alpha)\left(v_{k+1}-u_{k+1}\right)$. Se calcula $R^{2}\left(\lambda_{k+1}\right)$ e ir al paso 4 .

4) Se sustituye $k$ por $k+1$ e ir al paso 1 .

\section{Apéndice 2. Ajuste de las estructuras de tasas}

Udibonos: Vector de parámetros

$$
\left(\tau, \beta_{0}, \beta_{1}, \beta_{2}\right)=\left(\begin{array}{llll}
137.43673 & 0.04374 & -0.05026 & 0.08308
\end{array}\right) .
$$

\begin{tabular}{|c|c|c|c|c|c|}
\hline \multicolumn{2}{|c|}{ Datos Udibonos para el $28 / 01 / 2002$} & \multicolumn{3}{c|}{ Ajuste Udibonos } \\
\hline Plazo & Tasa Simple & Tasa Continua & Plazo & Tasa Simple & Tasa Continua \\
\hline 101 & 0.02720 & 0.02710 & 101 & 0.02719 & 0.02709 \\
185 & 0.03930 & 0.03891 & 185 & 0.03919 & 0.03880 \\
241 & 0.04850 & 0.04773 & 241 & 0.04670 & 0.04598 \\
297 & 0.04860 & 0.04765 & 297 & 0.04856 & 0.04761 \\
367 & 0.04870 & 0.04753 & 367 & 0.04995 & 0.04872 \\
423 & 0.05120 & 0.04972 & 423 & 0.05092 & 0.04945 \\
479 & 0.05170 & 0.05000 & 479 & 0.05150 & 0.04981 \\
549 & 0.05200 & 0.05004 & 549 & 0.05236 & 0.05037 \\
731 & 0.05250 & 0.04989 & 731 & 0.05289 & 0.05024 \\
913 & 0.05250 & 0.04929 & 913 & 0.05320 & 0.04990 \\
1109 & 0.05250 & 0.04866 & 1109 & 0.05356 & 0.04957 \\
2803 & 0.05450 & 0.04543 & 2803 & 0.05428 & 0.04527 \\
3265 & 0.05440 & 0.04422 & 3265 & 0.05460 & 0.04435 \\
\hline
\end{tabular}

Figura A.2.1. Tasa simple Udibinos.

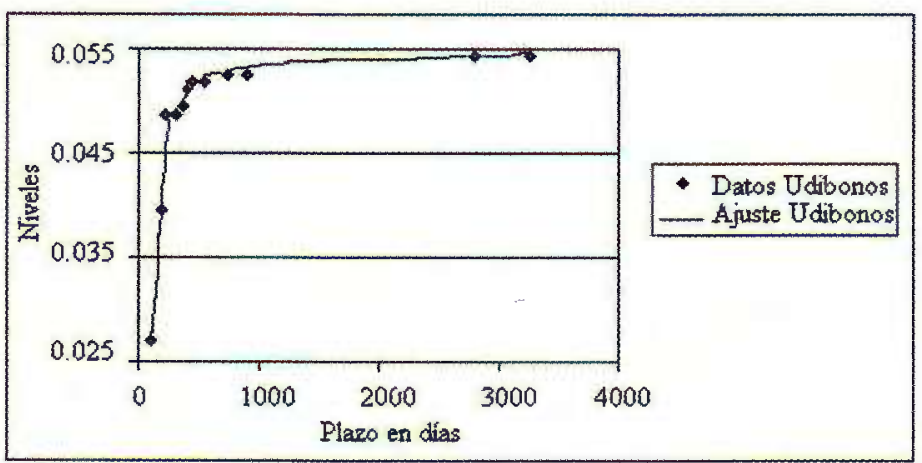


Libor: Vector de parámetros

$$
\left(\tau, \beta_{0}, \beta_{1}, \beta_{2}\right)=\left(\begin{array}{llll}
20.21242 & 0.02390 & -0.00346 & -0.01682
\end{array}\right) .
$$

\begin{tabular}{|c|c|c|c|c|c|}
\hline \multicolumn{3}{|c|}{ Datos Libor para el $28 / 01 / 2002$} & \multicolumn{3}{c|}{ Ajuste Libor } \\
\hline Plazo & Tasa Simple & Tasa Continua & Plazo & Tasa Simple & Tasa Continua \\
\hline 7 & 0.01828 & 0.01827 & 7 & 0.01827 & 0.01827 \\
28 & 0.01830 & 0.01829 & 28 & 0.01829 & 0.01828 \\
91 & 0.01870 & 0.01866 & 91 & 0.01868 & 0.01864 \\
182 & 0.02023 & 0.02012 & 182 & 0.02020 & 0.02010 \\
273 & 0.02243 & 0.02224 & 273 & 0.02241 & 0.02222 \\
365 & 0.02501 & 0.02470 & 365 & 0.02493 & 0.02462 \\
\hline
\end{tabular}

Figura A.2.2. Tasa simple Libor.

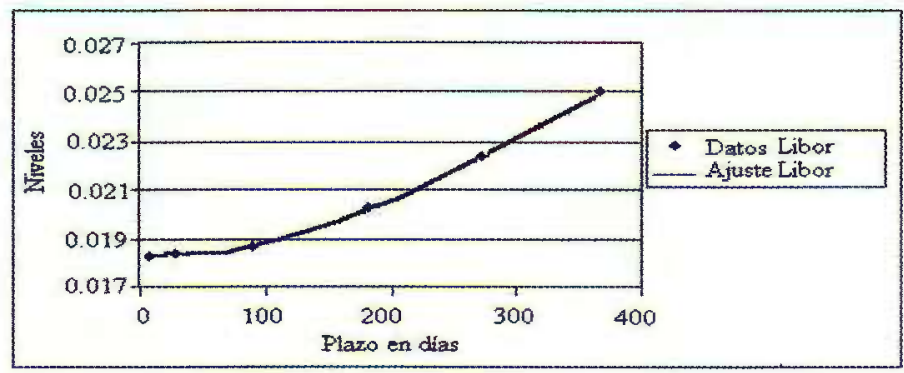

T-Bill: Vector de parámetros

$$
\left(\tau, \beta_{0}, \beta_{1}, \beta_{2}\right)=\left(\begin{array}{llll}
1261.98167 & 0.02546 & -0.01169 & 0.07020
\end{array}\right) .
$$

\begin{tabular}{|c|c|c|c|c|c|}
\hline \multicolumn{3}{|c|}{ Datos T-Bill para el 28/01/2002 } & \multicolumn{3}{c|}{ Ajuste T-Bill } \\
\hline Plazo & Tasa Simple & Tasa Continua & Plazo & Tasa Simple & Tasa Continua \\
\hline 91 & 0.01720 & 0.01716 & 91 & 0.01654 & 0.01651 \\
182 & 0.01852 & 0.01843 & 182 & 0.01879 & 0.01870 \\
730 & 0.03171 & 0.03073 & 730 & 0.03165 & 0.03068 \\
1825 & 0.04412 & 0.03982 & 1825 & 0.04375 & 0.03951 \\
10950 & 0.05465 & 0.03219 & 10950 & 0.05391 & 0.03191 \\
\hline
\end{tabular}

Figura A.2.3. Tasa simple T-Bill.

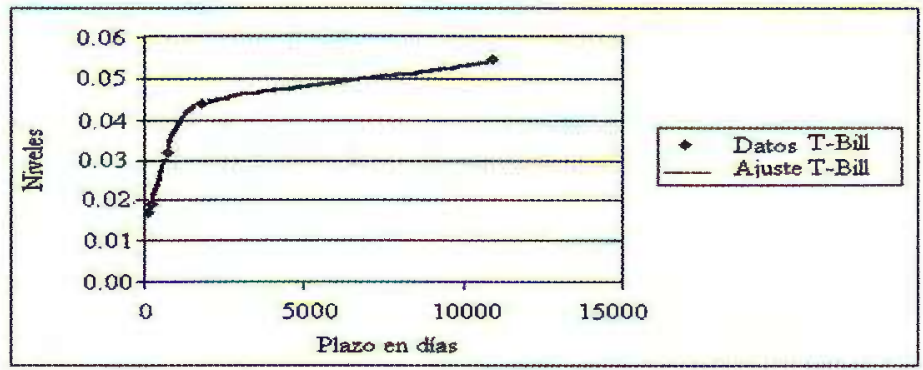


Apéndice 3. Histogramas de los parámetros

Udibonos

Figura A.3.1. Parámetro $\tau$.

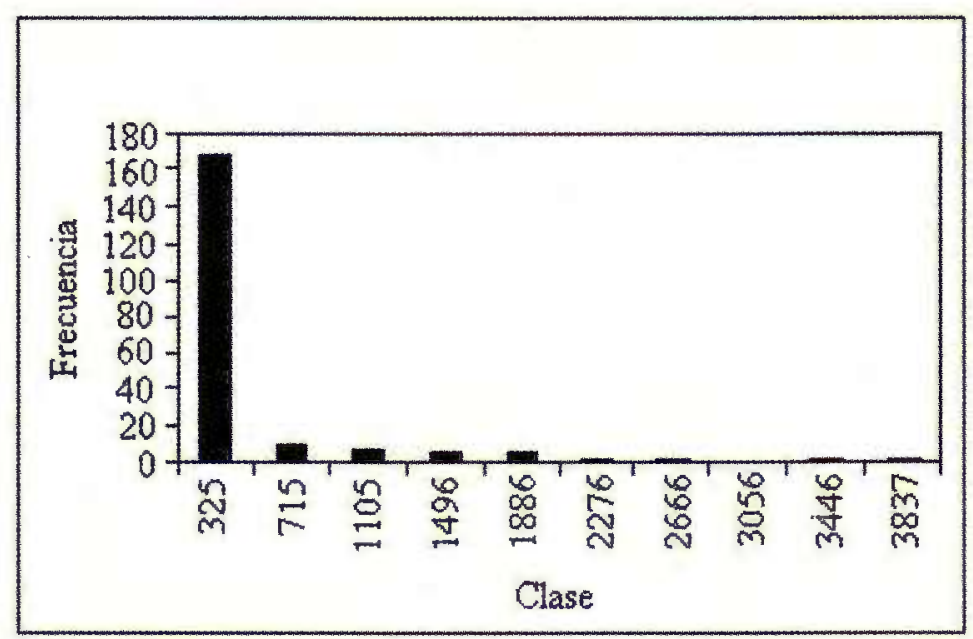

Figura A.3.2. Parámetro $\beta_{0}$.

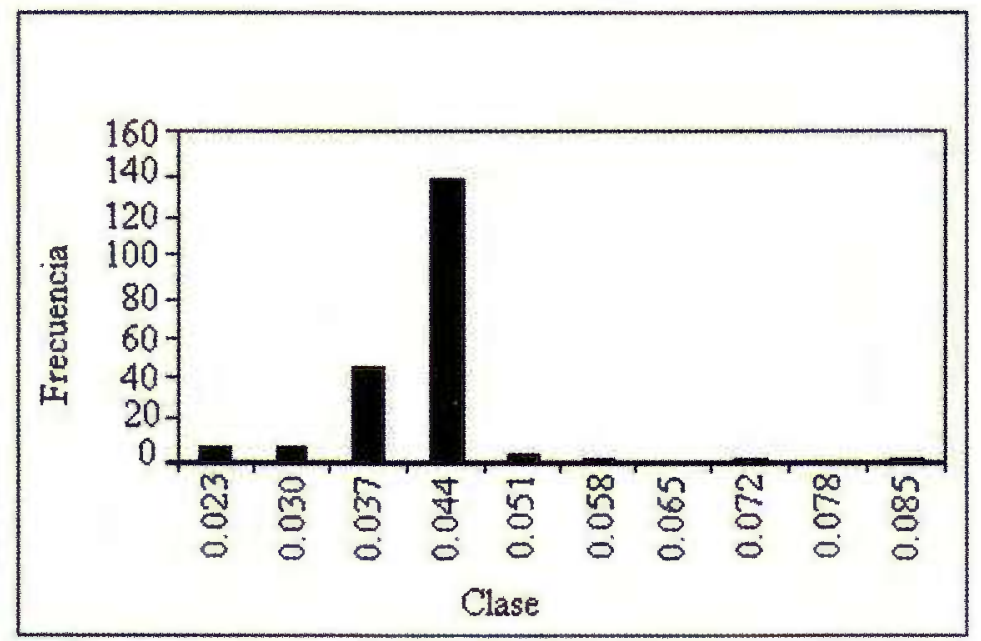


Figura A.3.3. Parámetro $\beta_{1}$.

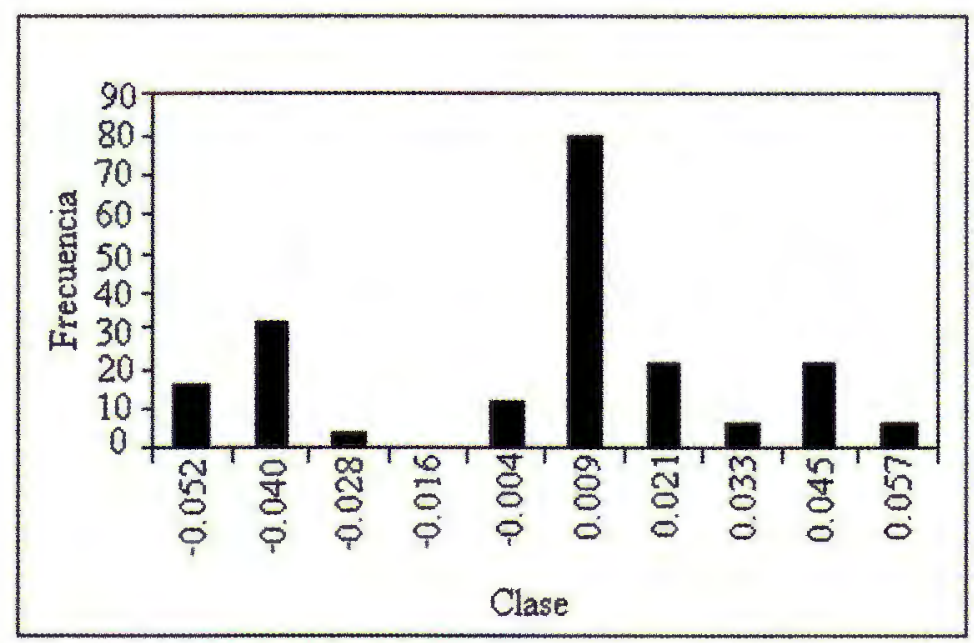

Figura A.3.4. Parámetro $\beta_{2}$.

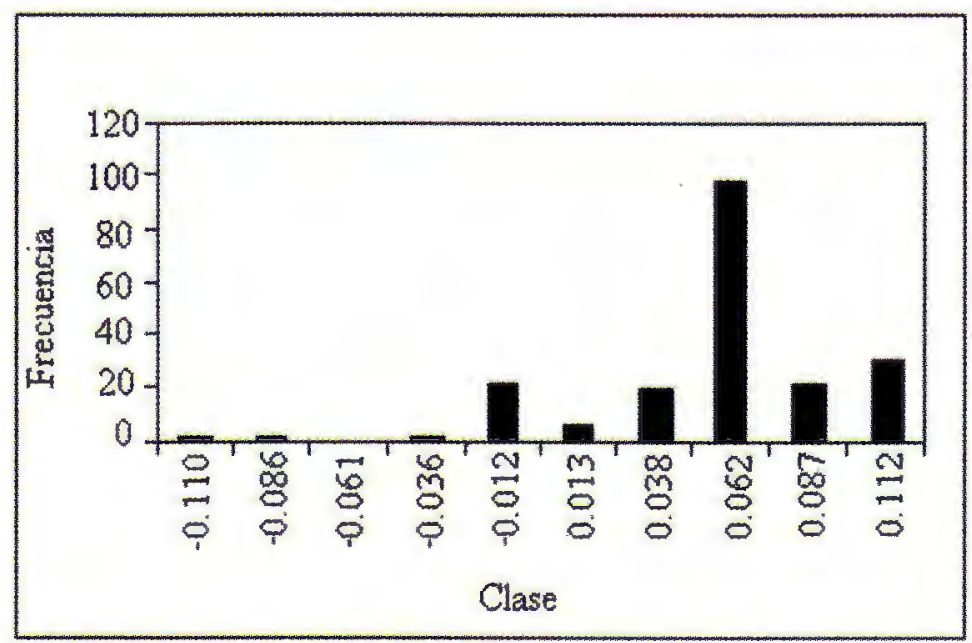


Libor

Figura A.3.5. Parámetro $\tau$.

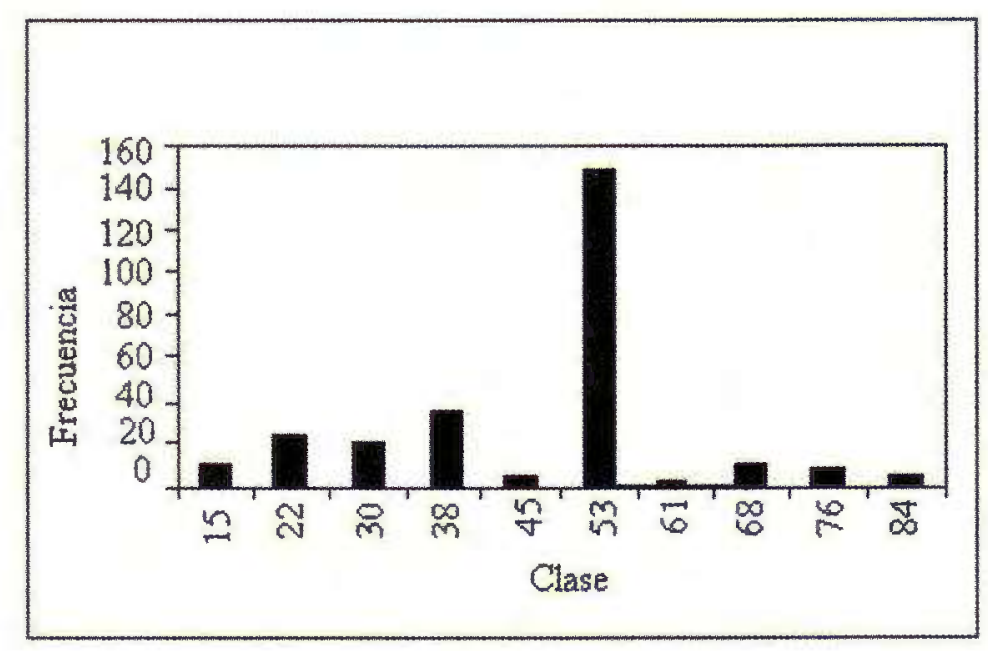

Figura A.3.6. Parámetro $\beta_{0}$.

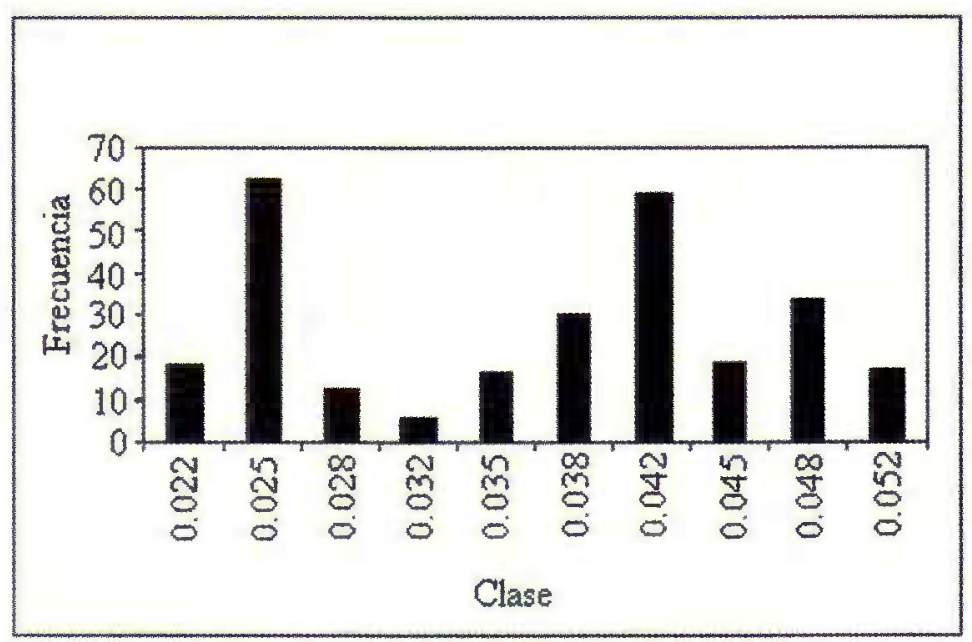


Figura A.3.7. Parámetro $\beta_{1}$.

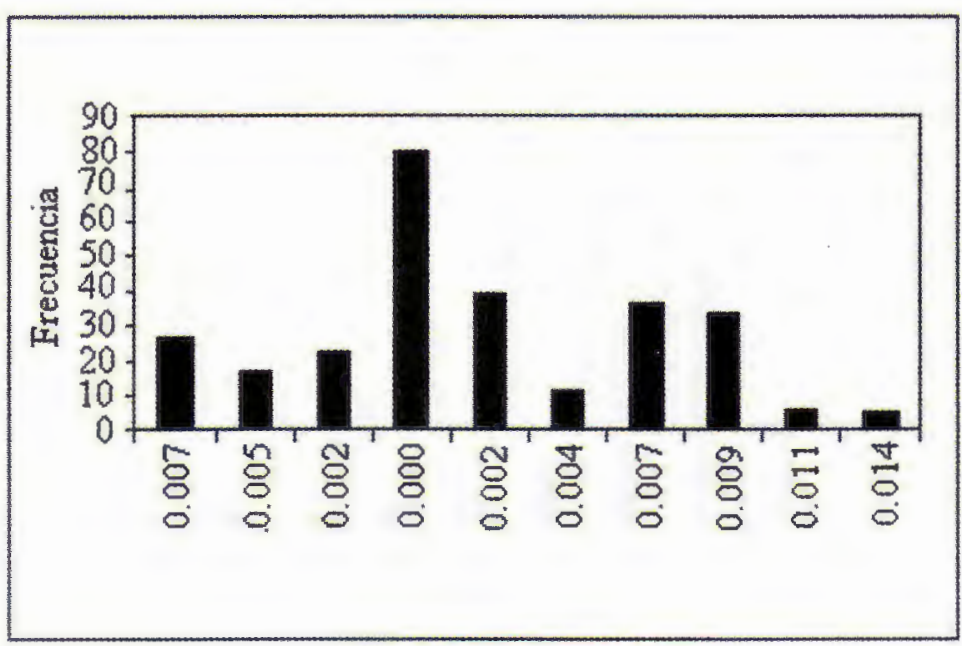

Figura A.3.8. Parámetro $\beta_{2}$.

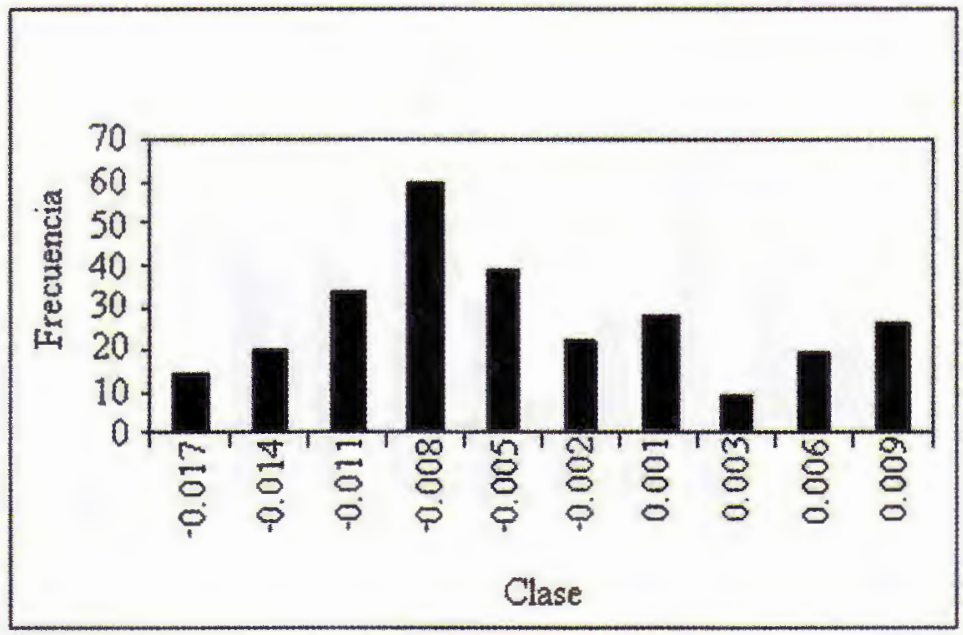


T-Bill

Figura A.3.9. Parámetro $\tau$.

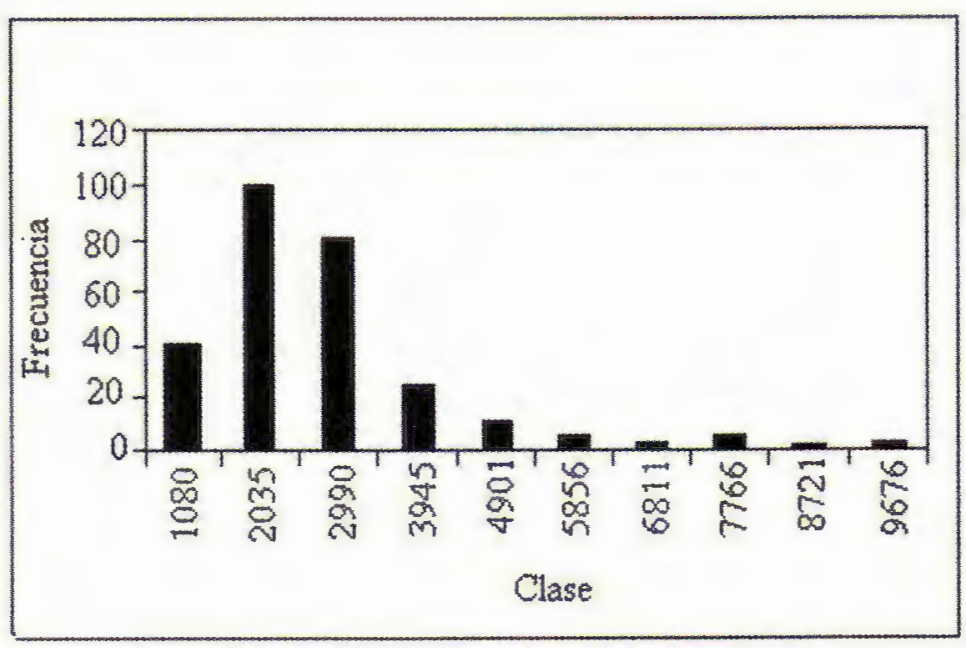

Figura A.3.10. Parámetro $\beta_{0}$.

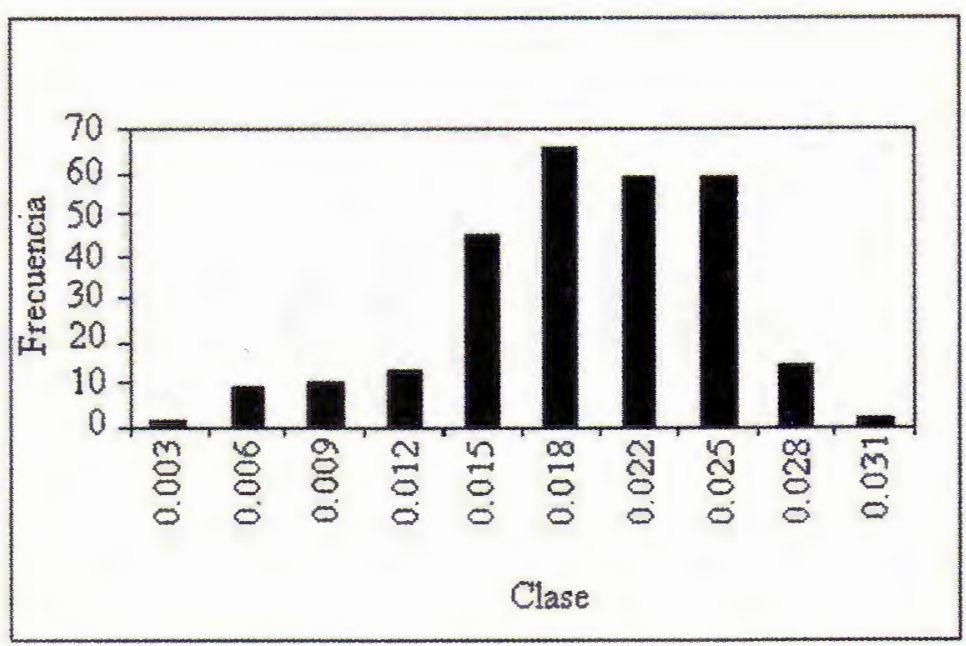


Figura A.3.11. Parámetro $\beta_{1}$.

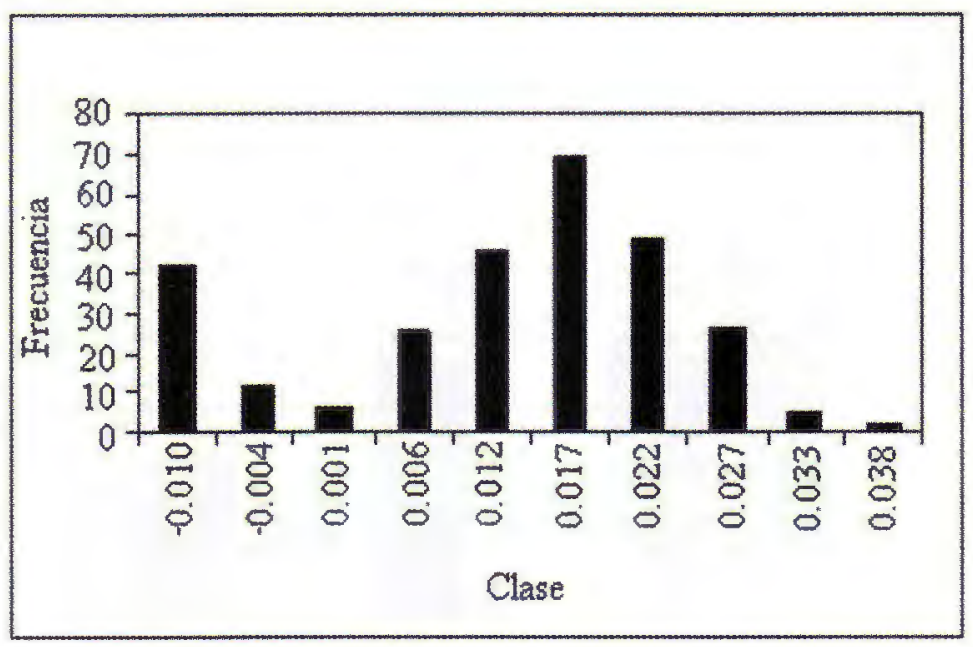

Figura A.3.12. Parámetro $\beta_{2}$.

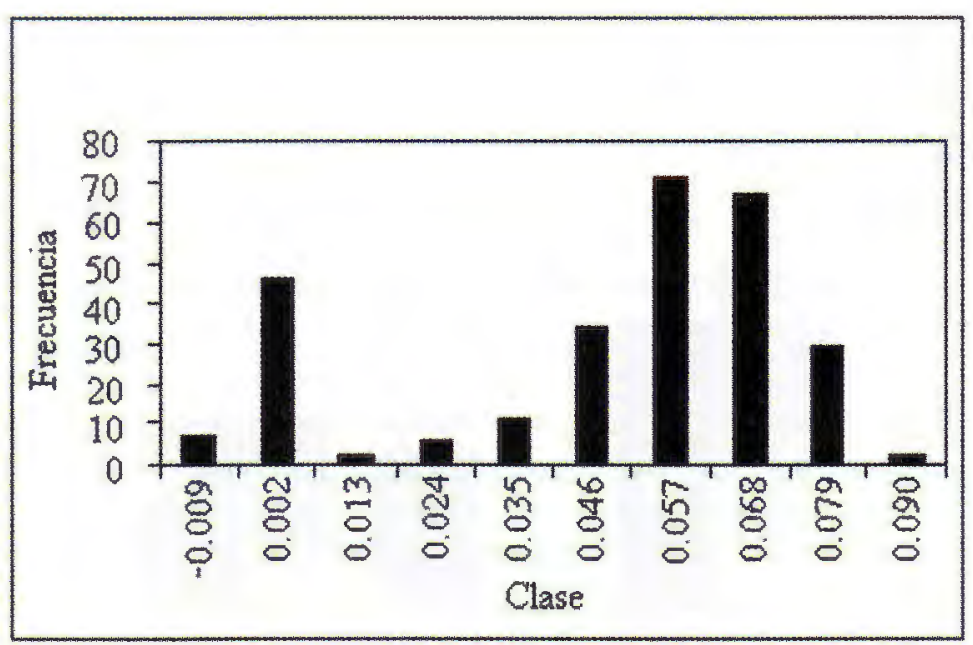




\section{Apéndice 4. Simulaciones}

Udibonos

Figura A.4.1. Forma de las curvas originales.

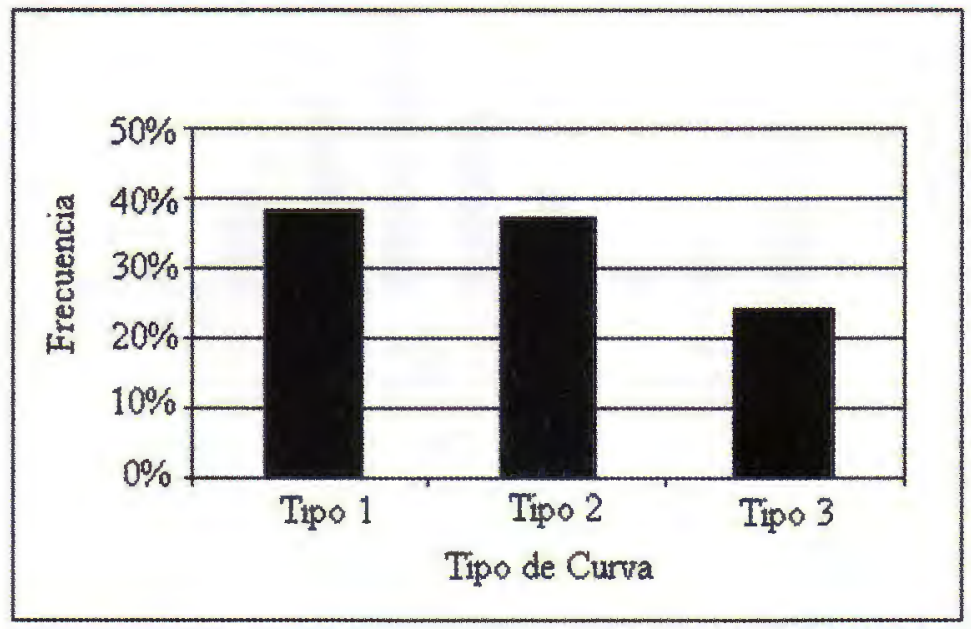

Figura A.4.2. Forma de las curvas generadas por la simulación.

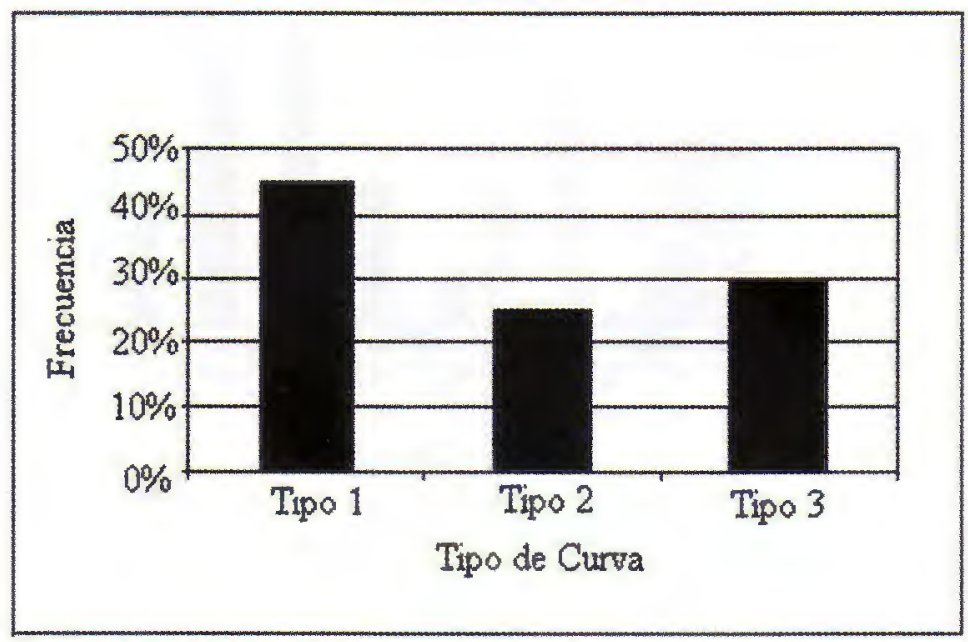


Libor

Figura A.4.3. Forma de las curvas originales.

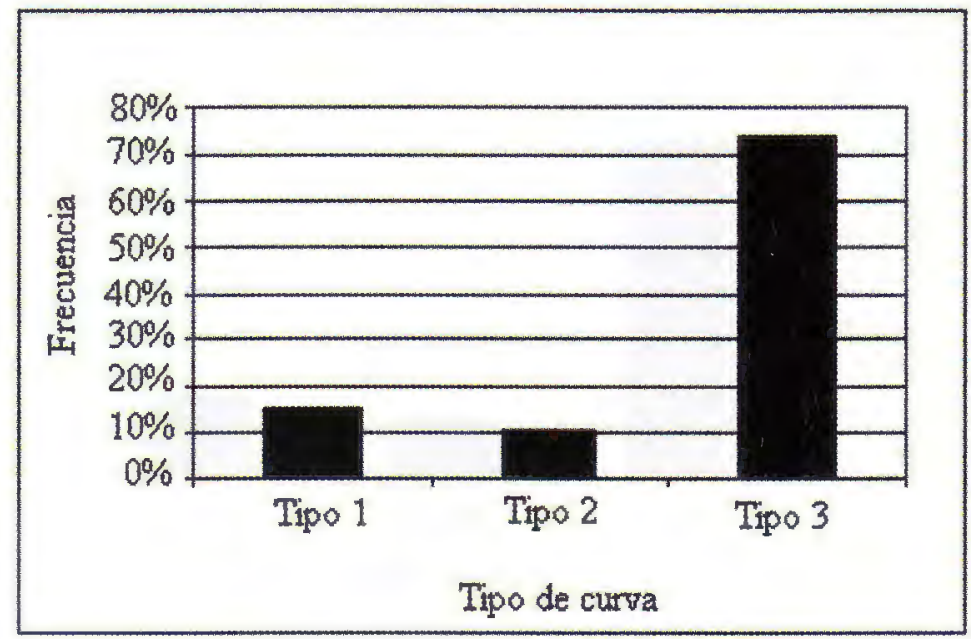

Figura A.4.4. Forma de las curvas generadas por la simulación.

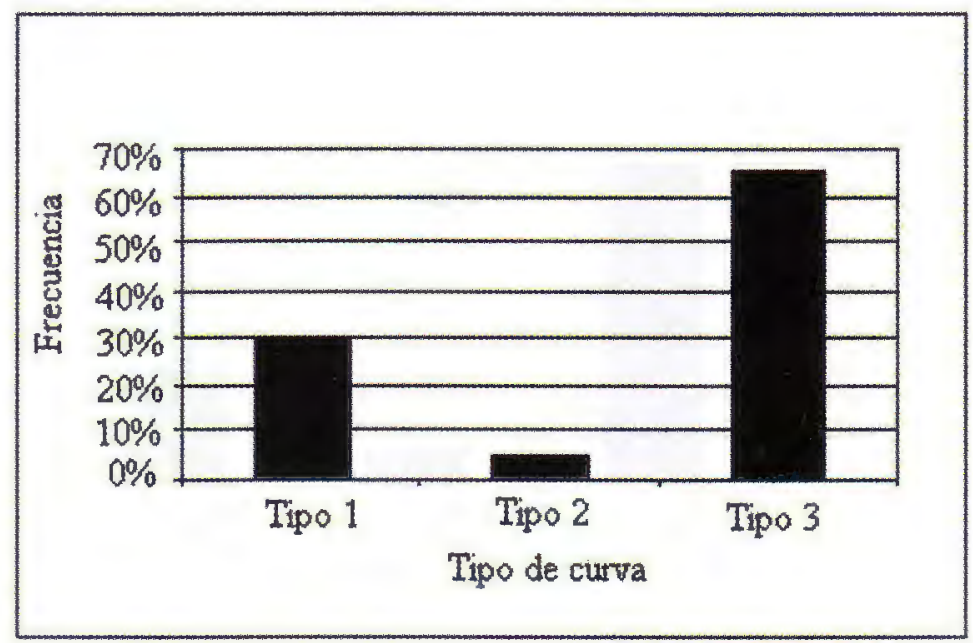


T-Bill

Figura A.4.5. Forma de las curvas originales.

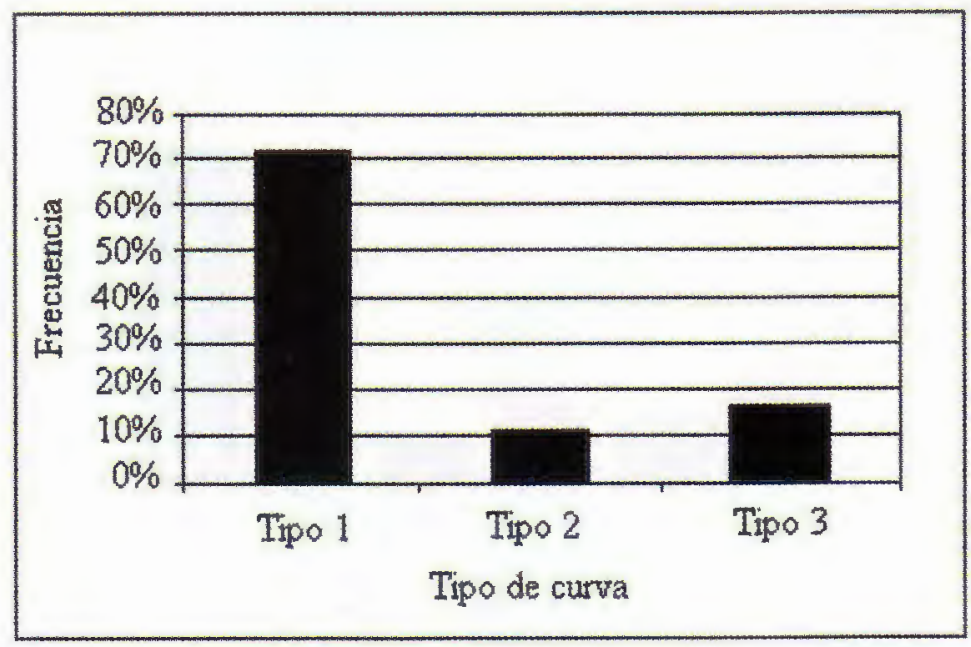

Figura A.4.6. Forma de las curvas generadas por la simulación.

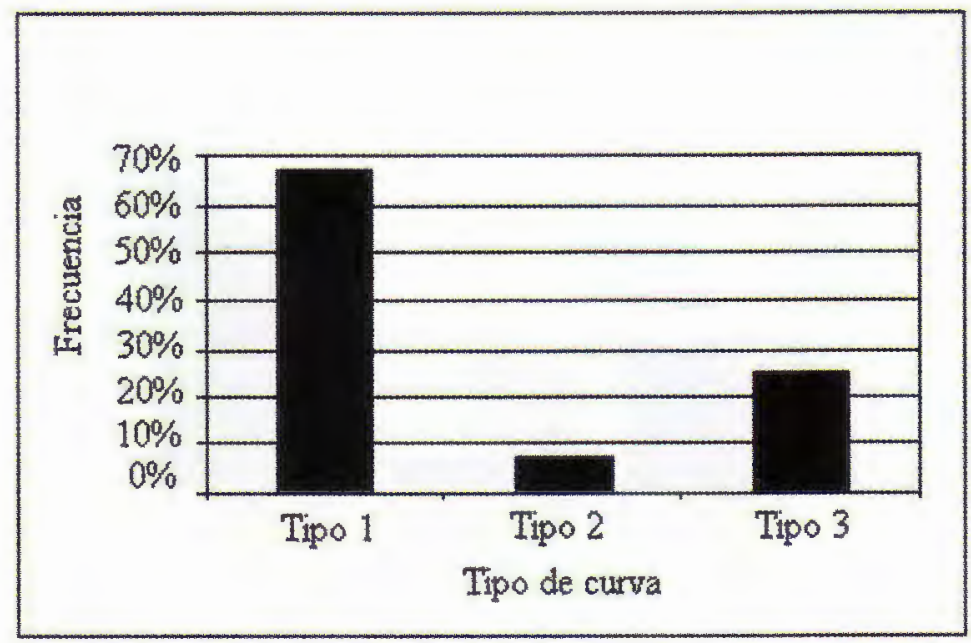




\section{Bibliografía}

Bliss, R. R. (1996). Testing Term Structure Estimation Methods. Working Paper 96-12a, Federal Reserve Bank of Atlanta.

Márquez Diez-Canedo, J. (1984). Fundamentos de Teoria de Optimización. Limusa, México. Bazaraa, M. S. and C. M. Shetty (1979). Nonlinear Programming, Theory and Algorithms. John Wiley \& Sons, USA.

J.P. Morgan and Reuters (1996). RiskMetrics-Technical Document. New York, Fourth Edition.

Nelson, C. R. and A. F. Siegel (1987). Parsimonious Modeling and Yield Curves. Journal of Business, 60, pp. 473-489.

Svensson, L. E. O. (1994). Estimating and Interpreting Forward Interest Rates: Sweden 1992-1994. Working Paper No. 4871, National Bureau of Economic Research. 